\title{
REVISÃo DE LITERATURA O que é comportamento de risco para transtornos alimentares em adolescentes?
}

\author{
What is adolescents' eating disorder risk behavior?
}

Greisse Viero da Silva Leal1', Sonia Tucunduva Philippi², Viviane Ozores Polacow³ , Táki Athanássios Cordás, Marle dos Santos Alvarenga'

\section{RESUMO}

Objetivo: Caracterizar comportamento de risco para transtornos alimentares (TA) e sua frequência entre adolescentes em uma revisão da literatura nacional e internacional. Métodos: Foi realizada uma busca bibliográfica por meio de uma revisão integrativa nas bases de dados PubMed (US National Library of Medicine) e Lilacs e no portal SciELO, utilizando-se os descritores relacionados à "eating disorder risk behavior". Foram selecionados artigos publicados nos últimos 10 anos, nos idiomas português, espanhol e inglês, e especificamente com adolescentes. Foram avaliados 76 artigos e analisados a nomenclatura e os instrumentos utilizados para avaliar comportamento de risco para TA e sua prevalência. Resultados: Encontrou-se uma série de termos para avaliar risco para TA. A metodologia mais utilizada foi a de questionários e escalas, destacando-se o EAT-26 ou 40 e o BITE, dentre os mais frequentes; a prevalência de risco variou de $0,24 \%$ a $58,4 \%$. Conclusão: Diferentes nomenclaturas e

\section{Palavras-chave}

Transtornos da alimentação, adolescente, risco.

\section{Keywords}

Eating disorders, adolescent, risk. instrumentos são utilizados para avaliar comportamento de risco para TA entre adolescentes, com grande amplitude nos resultados de prevalência. Maior padronização de termos e metodologia de avaliação permitiriam melhor comparação entre estudos epidemiológicos em diferentes localidades.

\section{ABSTRACT}

Objective: To characterize eating disorder (ED) risk behavior and its prevalence among adolescents in a review of national and international literature. Methods: We carried out an integrative review on PubMed (U.S. National Library of Medicine), Lilacs and SciELO, using descriptors related to "eating disorder risk behavior". Articles published in the last 10 years, in Portuguese, Spanish and English, and specifically with adolescents were selected. The nomenclature and instruments used to assess eating disorder risk behaviors and its frequency were evaluated and analyzed in 76 articles. Results: Several terms to assess ED risk behavior were found; questionnaires and scales were used, mainly EAT-26 or 40 and BITE. The prevalence of risk ranged from $0.24 \%$ to $58.4 \%$. Conclusion: Different names and instruments are used to assess ED risk behavior among adolescents, and there is a wide variation in the prevalence. Standardization of terms and evaluation methodology might enhance a better comparison of epidemiological studies in different locations.

1 Universidade de São Paulo (USP), Faculdade de Saúde Pública (FSP); Instituto de Psiquiatria, Hospital das Clínicas, Faculdade de Medicina da USP (IPq-HC-FMUSP), Ambulatório de Bulimia e Transtornos Alimentares (Ambulim), Programa de Transtornos Alimentares.

2 FSP/USP, Departamento de Nutrição.

3 USP, Escola de Educação Física e Esporte (EEFE); IPq-HC-FMUSP, Ambulatório de Bulimia e Transtornos Alimentares (Ambulim), Programa de Transtornos Alimentares.

4 FMUSP, Departamento de Psiquiatria; IPq-HC-FMUSP, Ambulim, Programa de Transtornos Alimentares.

Endereço para correspondência: Greisse Viero da Silva Leal

Faculdade de Saúde Pública, Universidade de São Paulo, Departamento de Nutrição

Av. Doutor Arnaldo, 715

01246-904 - São Paulo, SP, Brasil

Email: greisse@usp.br 


\section{INTRODUÇÃO}

Os transtornos alimentares (TA) têm critérios diagnósticos baseados em características psicológicas, comportamentais e fisiológicas'. Caracterizam-se por severas perturbações no comportamento alimentar e imagem corporal, e seus critérios diagnósticos são estabelecidos pela Organização Mundial de Saúde, no Código Internacional de Doenças (CID-10), e pela Associação de Psiquiatria Americana, no Manual de Estatísticas de Doenças Mentais (DSM-IV)².

Os quadros mais conhecidos são a anorexia nervosa (AN) e a bulimia nervosa (BN). Quando todos os critérios diagnósticos não são preenchidos para uma dessas síndromes, utiliza-se o diagnóstico de Transtorno Alimentar Sem Outra Especificação (TASOE) $)^{3}$ e, dentre eles, destaca-se o transtorno da compulsão alimentar periódica (TCAP).

Transtornos alimentares são mais frequentes entre muIheres adolescentes ou adultas jovens, afetando 3,2\% das mulheres com idade entre 18 e 30 anos, e com uma relação homem:mulher variando de 1:6 a 1:101,4. Estudos sobre a prevalência de AN entre mulheres na adolescência tardia e início da idade adulta verificam taxas de 0,5\% a 1\%, segundo o DSM-IV. Com relação à BN, pelo menos $90 \%$ dos indivíduos são mulheres, e a sua prevalência entre adolescentes e adultas jovens é de aproximadamente $1 \%$ a $3 \% 3,5$.

Os TA têm uma etiologia multifatorial (diferentes fatores interagindo para o desenvolvimento e perpetuação da doença) ${ }^{4}$. Há, portanto, dificuldade na realização de estudos de incidência e prevalência, pois são necessárias avaliações e abordagens que contemplem os vários aspectos envolvidos em sua gênese e manutenção. Assim, pesquisas sobre comportamento de risco podem dar um indício do problema em determinado local e população 2,6.

Embora os TA tenham definição e critérios diagnósticos padronizados internacionalmente, não há a mesma preocupação em relação àquilo que se denomina "comportamentos de risco para TA". Esses são avaliados considerando-se basicamente a presença dos sintomas clássicos dos quadros: restrição alimentar (hábito de fazer dietas restritivas ou jejum), compulsão alimentar e práticas purgativas (uso de laxantes, diuréticos e vômitos autoinduzidos) ${ }^{7-10}$.

Diferentes nomenclaturas podem ser adotadas para avaliar esses mesmos aspectos, por exemplo, comportamento alimentar anormal' ${ }^{11}$, suspeita de transtornos alimentares ${ }^{12}$, risco de transtornos alimentares ${ }^{13}$, comportamentos de risco para transtornos alimentares ${ }^{7}$, sintomas de transtornos alimentares ${ }^{14}$, atitudes alimentares anormais ${ }^{15}$, padrões alimentares anormais ${ }^{16}$. Outra terminologia utilizada é o "comer transtornado", definido pela Associação de Psiquiatria Americana e pela Associação Dietética Americana como todo espectro dos problemas alimentares, da "simples dieta" aos TA clássicos ${ }^{1.4}$. Nesse conceito podem estar incluídas também práticas não saudáveis para controle do peso, tais como fazer jejum ou comer muito pouca comida, pular refeições, usar de substitutos de alimentos como suplementos ou shakes, tomar remédios para emagrecer ou fumar mais cigarros ${ }^{17}$.

Dessa forma, para conhecer o "estado da arte" da presença de TA ou comportamentos de risco numa determinada população e local, os pesquisadores necessitam usar uma gama de nomenclaturas possíveis. Considerando-se que os comportamentos de risco para TA ocorrem anteriormente aos distúrbios clássicos e que podem levar a prejuízos físicos e emocionais, isolamento social, insatisfação corporal - além do risco de evoluir para quadros completos -, justifica-se a sua identificação precoce $e^{9,18,19}$.

Comportamentos de risco para TA são avaliados habitualmente por meio de questionários padronizados, tais como o Eating Attitudes Test (EAT), Eating Disorder Inventory (EDI), Eating Disorder Examination versão questionário (EDE-Q), Binge Eating Scale (BES), Bulimia Test (BULIT), Bulimic Investigatory Test, Edinburgh (BITE), entre outros, que surgiram pela necessidade de sistematizar os métodos de estudos de TA após o estabelecimento de seus critérios diagnósticos. Alguns desses instrumentos abrangem todos os tipos de TA, enquanto outros são mais específicos para um ou outro transtorno isoladamente ${ }^{6}$.

No Brasil, os estudos sobre TA e/ou comportamentos de risco são ainda, em sua maioria, locais e pontuais $2,7,10,14,20-25$, sem uma padronização de nomenclatura e metodologia de avaliação. Nesse contexto, o objetivo deste estudo foi caracterizar o comportamento de risco para transtornos alimentares, incluindo os instrumentos utilizados para identificação, a nomenclatura adotada e sua prevalência ou frequência entre adolescentes em uma revisão integrativa da literatura nacional e internacional.

\section{MÉTODOS}

Foi realizada uma busca bibliográfica por meio de revisão integrativa ${ }^{26}$ nas bases de dados PubMed (US National Library of Medicine) e Lilacs (Literatura Latino-Americana e do Caribe em Ciências da Saúde) e no portal SciELO (The Scientific Electronic Library Online), utilizando-se os descritores agrupados de diferentes formas, conforme sentenças descritas no quadro 1. Como as expressões "comportamento de risco para transtornos alimentares" ou "eating disorder risk behavior" não se caracterizavam como descritores em Ciências da Saúde e, portanto, se mostraram insuficientes para localização de trabalhos, uma série de descritores possivelmente relacionados ao tema foi utilizada, incluindo o nome de alguns instrumentos padronizados e de alguns autores de referência.

\section{Quadro 1}

Os critérios adotados para inclusão dos artigos no estudo foram: a amostra deveria incluir apenas adolescentes (10- 
19 anos); os estudos deveriam ser originais - transversais e/ ou longitudinais; deveriam avaliar a presença de comportamentos de risco para transtornos alimentares, tais como compulsão alimentar, uso de laxantes e/ou diuréticos, vômito autoinduzido, prática de dieta restritiva, além de práticas não saudáveis para controle do peso (independentemente da nomenclatura utilizada); e apresentar a frequência ou a prevalência desses comportamentos.

Quadro 1 - Estratégias de busca utilizadas em cada base de dados a partir das palavras-chave

\begin{tabular}{|c|c|c|c|}
\hline Conjuntos & PubMed & $\begin{array}{c}\text { Número } \\
\text { de artigos } \\
\text { encontrados }\end{array}$ & $\begin{array}{c}\text { Número } \\
\text { de artigos } \\
\text { selecionados }\end{array}$ \\
\hline 1 & $\begin{array}{l}\text { Eating disorders AND adolescent } \\
\text { AND risk }\end{array}$ & 142 & 27 \\
\hline 2 & $\begin{array}{c}\text { Disordered eating AND adolescent } \\
\text { AND risk }\end{array}$ & 58 & 6 \\
\hline 3 & EAT-26 AND adolescent AND risk & 17 & 2 \\
\hline 4 & BITE AND adolescent AND risk & 2 & 2 \\
\hline 5 & EDI AND adolescent AND risk & 12 & 0 \\
\hline 6 & EDE AND adolescent AND risk & 4 & 0 \\
\hline 7 & $\begin{array}{l}\text { Hay, Phillipa J (author) AND adolescent } \\
\text { (title/abstract) AND eating disorders } \\
\text { (title/abstract) OR disordered eating } \\
\text { (title/abstract) }\end{array}$ & 462 & 19 \\
\hline 8 & $\begin{array}{c}\text { Hay P AND prevalence of eating } \\
\text { behaviors }\end{array}$ & 7 & 0 \\
\hline 9 & Hay P (author) & 54 & 0 \\
\hline Conjuntos & SCIELO & $\begin{array}{c}\text { Número } \\
\text { de artigos } \\
\text { encontrados }\end{array}$ & $\begin{array}{c}\text { Número } \\
\text { de artigos } \\
\text { selecionados }\end{array}$ \\
\hline 1 & $\begin{array}{c}\text { Transtornos alimentares AND } \\
\text { adolescentes }\end{array}$ & 8 & 4 \\
\hline 2 & EAT AND adolescente & 7 & 1 \\
\hline 3 & BITE AND adolescente & 5 & 1 \\
\hline Conjuntos & Lilacs & $\begin{array}{c}\text { Número } \\
\text { de artigos } \\
\text { encontrados }\end{array}$ & $\begin{array}{c}\text { Número } \\
\text { de artigos } \\
\text { selecionados }\end{array}$ \\
\hline 1 & $\begin{array}{c}\text { Transtornos da alimentação AND } \\
\text { adolescente AND risco }\end{array}$ & 34 & 9 \\
\hline 2 & EAT-26 AND adolescente AND risco & 23 & 2 \\
\hline 3 & BITE AND transtornos alimentares & 8 & 2 \\
\hline 4 & EDI AND transtornos alimentares & 11 & 0 \\
\hline 5 & EDE AND transtornos alimentares & 1 & 1 \\
\hline Conjuntos & Google acadêmico & $\begin{array}{c}\text { Número } \\
\text { de artigos } \\
\text { encontrados }\end{array}$ & $\begin{array}{c}\text { Número } \\
\text { de artigos } \\
\text { selecionados }\end{array}$ \\
\hline 1 & "Phillipa Hay" eating disorders & 141 & 0 \\
\hline
\end{tabular}

Como critérios de exclusão de artigos foram utilizados: artigos de revisão; pesquisas focadas em grupos específicos (como portadores de diabetes mellitus, atletas e bailarinos); artigos com indivíduos com AN e BN já diagnosticados; pesquisas focalizadas na associação de fatores de risco para TA (influência da mídia, família, amigos etc.), ou comportamento alimentar, ou prevenção e tratamento de TA; e artigos com foco em outras psicopatologias, fatores genéticos, compulsão por bebidas, orientação sexual, insatisfação corporal, síndrome do comer noturno, uso de substâncias, abuso sexual, body checking, complicações clínicas, saúde oral, refeições em família e teasing - e que não apresentavam a frequência ou a prevalência de comportamento de risco para TA.

\section{RESULTADOS}

Foram identificadas 996 publicações de acordo com as estratégias de busca definidas. No caso da base de dados PubMed, pela extensão dos resultados, foram adotados limites: artigos publicados nos últimos 10 anos; idiomas português, espanhol e inglês; faixa etária da adolescência; e palavras-chave no título ou resumo.

Após avaliação dos títulos e resumos, utilizando-se os critérios de inclusão e exclusão, 190 artigos foram considerados potencialmente elegíveis para inclusão nesta revisão. Após a leitura dos artigos, 74 foram excluídos por terem sido realizados com grupos específicos ou com indivíduos adultos e por se tratar de revisões, 36 eram repetidos e 2 não estavam disponíveis, sendo incluídos na presente revisão 76 artigos (Figura 1).

Dos artigos selecionados, que avaliaram especificamente comportamento de risco para TA em adolescentes, foram destacados a autoria, o ano de publicação, a população de estudo, o país de origem, a nomenclatura utilizada e o instrumento utilizado para avaliar comportamento de risco para TA e sua frequência ou prevalência. Podem ser observados nas tabelas 1, 2, 3 e 4 os trabalhos encontrados no Brasil; América Latina; Estados Unidos e Canadá; Europa, Ásia, África e Austrália, respectivamente.

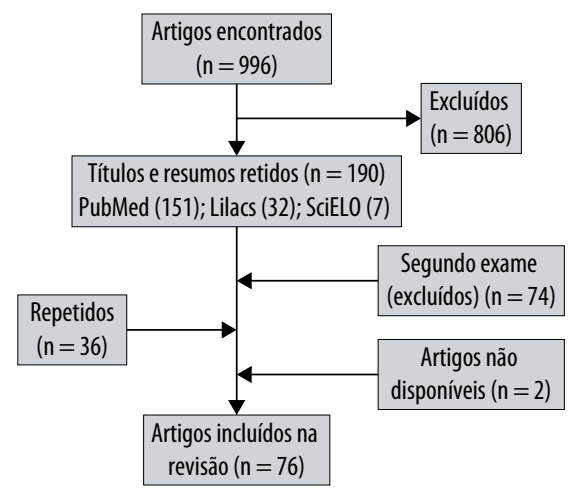

Figura 1. Etapas de seleção dos artigos incluídos na revisão integrativa. 
Tabela 1 - Estudos brasileiros realizados sobre comportamentos de risco para transtornos alimentares entre adolescentes

\begin{tabular}{|c|c|c|c|c|c|c|}
\hline Autor/Ano & População de estudo & Idade (anos) & Estado/País & Questionário & Nomenclatura utilizada & Prevalência/Frequência* \\
\hline Alves et al., $2008^{20}$ & 1.148 meninas & $10-19$ & Florianópolis, Brasil & Eating Attitudes Test (EAT-26) & $\begin{array}{l}\text { Sintomas de anorexia } \\
\text { nervosa }\end{array}$ & $15,6 \%$ \\
\hline Dunker et al., $2009^{21}$ & 183 meninas & $15-18$ & São Paulo, Brasil & Eating Attitudes Test (EAT-26) & $\begin{array}{l}\text { Comportamento de } \\
\text { risco para TA }\end{array}$ & $\begin{array}{l}\text { Escola particular } \\
34,3 \% * \\
\text { Escola pública } 28,4 \% *\end{array}$ \\
\hline Ferreira e Veiga, $2008^{7}$ & 561 & $12-19$ & Rio de Janeiro, Brasil & $\begin{array}{l}\text { Questionário adaptado de Hay }(1998)^{40} \text { : } \\
\text { questões sobre compulsão, dieta } \\
\text { restritiva, uso de laxante, diurético ou } \\
\text { vômito autoinduzido }\end{array}$ & $\begin{array}{l}\text { Comportamento de } \\
\text { risco para TA (eating } \\
\text { disorder risk behavior) }\end{array}$ & $\begin{array}{l}\text { Compulsão: } 37,3 \% \\
\text { Dieta restritiva: } 24,7 \% \\
\text { Laxante: } 2,1 \% \\
\text { Diurético: } 1,6 \% \\
\text { Vômito autoinduzido: } \\
\text { 1,4\% }\end{array}$ \\
\hline Gomes et al., $2010^{14}$ & 300 meninas & $10-15$ & Paraná, Brasil & $\begin{array}{l}\text { Bulimic Investigatory Test Edinburgh } \\
\text { (BITE) } \\
\text { Eating Attitudes Test (EAT-26) }\end{array}$ & Sintomas de TA & $\begin{array}{l}\text { BITE: } 4,2 \%{ }^{*} \\
\text { EAT- } 26: 3,6 \% *\end{array}$ \\
\hline Sampei et al., $2009^{23}$ & 544 meninas & $10-17$ & São Paulo, Brasil & Eating Attitudes Test (EAT-26) & $\begin{array}{l}\text { Atitudes alimentares } \\
\text { (eating attitudes) }\end{array}$ & $\begin{array}{l}\text { Japonesas } \\
\text { Pré-menarca: } 4,9 \%^{*} \\
\text { Pós-menarca: } 12,7 \%^{*} \\
\text { Caucasianas } \\
\text { Pré-menarca: } 26,7 \%^{*} \\
\text { Pós-menarca: } 19,4 \%^{*}\end{array}$ \\
\hline $\begin{array}{l}\text { Souza-Kaneshima, } \\
2008^{24}\end{array}$ & 187 & $15-19$ & Paraná, Brasil & $\begin{array}{l}\text { Bulimic Investigatory Test Edinburgh } \\
\text { (BITE) }\end{array}$ & $\begin{array}{l}\text { Comportamentos } \\
\text { favoráveis ao } \\
\text { desenvolvimento da } \\
\text { bulimia nervosa }\end{array}$ & $\begin{array}{l}\text { Comportamento } \\
\text { alimentar compulsivo: } \\
3,74 \%^{*} \\
\text { Comportamento } \\
\text { alimentar não usual: } \\
39,04 \%^{*}\end{array}$ \\
\hline Vale et al., $2011^{25}$ & 652 meninas & $14-20$ & Fortaleza, Brasil & $\begin{array}{l}\text { Bulimic Investigatory Test Edinburgh } \\
\text { (BITE) }\end{array}$ & $\begin{array}{l}\text { Comportamentos } \\
\text { de risco para TA (risk } \\
\text { behaviors for eating } \\
\text { disorders) }\end{array}$ & $25,2 \%$ \\
\hline Vilela et al., $2004^{10}$ & 1.807 & $7-19$ & Minas Gerais, Brasil & $\begin{array}{l}\text { Bulimic Investigatory Test Edinburgh } \\
\text { (BITE) } \\
\text { Eating Attitudes Test (EAT-26) }\end{array}$ & $\begin{array}{l}\text { Possíveis TA e } \\
\text { comportamento } \\
\text { alimentar inapropriados } \\
\text { (possible eating } \\
\text { disorders and } \\
\text { inappropriate eating } \\
\text { behaviors) }\end{array}$ & $\begin{array}{l}\text { BITE: } 1,1 \% * \\
\text { EAT-26: } 13,3 \% *\end{array}$ \\
\hline
\end{tabular}

Tabela 2 - Descrição de estudos realizados na América Latina sobre comportamentos de risco para transtornos alimentares em adolescentes

\begin{tabular}{|c|c|c|c|c|c|c|}
\hline Autor/Ano & População de estudo & Idade (anos) & Estado/País & Metodologia & Nomenclatura utilizada & Prevalência/Frequência* \\
\hline $\begin{array}{l}\text { Barriguete-Meléndez et } \\
\text { al., } 2009^{11}\end{array}$ & 25.166 & $10-19$ & México & $\begin{array}{l}\text { Perguntas próprias: The } \\
\text { Brief Questionnaire for } \\
\text { Risky Eating Behaviors } \\
\text { (10 itens que avaliam } \\
\text { comportamento } \\
\text { alimentar e medo } \\
\text { de ganhar peso nos } \\
\text { últimos } 3 \text { meses) }\end{array}$ & $\begin{array}{l}\text { Comportamento } \\
\text { alimentar anormal } \\
\text { (abnormal eating } \\
\text { behaviors) }\end{array}$ & $0,8 \%$ \\
\hline Bay et al., $2005^{12}$ & 1.971 & $10-19$ & Argentina & $\begin{array}{l}\text { Eating Disorder } \\
\text { Examination } \\
\text { Questionnaire (EDE-Q) }\end{array}$ & Suspeita de TA & $19,2 \%$ \\
\hline Behar et al., $2007^{27}$ & 296 & Alunos do ensino médio & Vina del Mar, Chile & $\begin{array}{l}\text { Eating Attitudes Test } \\
\text { (EAT-40) }\end{array}$ & Risco de TA & $12 \% *$ \\
\hline Correa et al., $2006^{28}$ & 1.050 & $11-19$ & Santiago, Chile & $\begin{array}{l}\text { Eating Disorder } \\
\text { Inventory (EDI-2) }\end{array}$ & Risco de TA & $8,3 \%^{*}$ \\
\hline $\begin{array}{l}\text { González e Viveros, } \\
2009^{29}\end{array}$ & 298 & $13-14$ & Veracruz, México & $\begin{array}{l}\text { Eating Attitudes Test } \\
\text { (EAT-40) }\end{array}$ & Risco de TA & $8 \% *$ \\
\hline
\end{tabular}




\begin{tabular}{|c|c|c|c|c|c|c|}
\hline Autor/Ano & População de estudo & Idade (anos) & Estado/País & Metodologia & Nomenclatura utilizada & Prevalência/Frequência* \\
\hline Granillo et al., $2005^{30}$ & 1.866 meninas & $11-20$ & $\begin{array}{l}\text { México, Cuba e Porto } \\
\text { Rico }\end{array}$ & $\begin{array}{l}\text { IMC < } 17 \mathrm{~kg} / \mathrm{m}^{2} \text {, } \\
\text { amenorreia (há mais } \\
\text { de } 2 \text { meses), restrição } \\
\text { dietética e sintomas } \\
\text { bulímicos (compulsão, } \\
\text { exercício excessivo, } \\
\text { vômito auto- induzido, } \\
\text { uso de remédios para } \\
\text { emagrecer e uso de } \\
\text { laxantes) }\end{array}$ & $\begin{array}{l}\text { Risco para TA (risk for } \\
\text { eating disorders) }\end{array}$ & $\begin{array}{l}\mathrm{IMC}<17 \mathrm{~kg} / \mathrm{m}^{2}: 2,5 \% \\
\text { Amenorreia: } 5,5 \% \\
\text { Restrição alimentar: } \\
53,3 \% \\
\text { Sintomas bulímicos: } \\
1,9 \%\end{array}$ \\
\hline $\begin{array}{l}\text { Mancilla-Díaz et al., } \\
2009^{31}\end{array}$ & 615 & $13-16$ & México & $\begin{array}{l}\text { Eating Attitudes Test } \\
\text { (EAT-40) } \\
\text { Bulimia Test (BULIT) }\end{array}$ & $\begin{array}{l}\text { Comportamentos } \\
\text { alimentares } \\
\text { transtornados } \\
\text { (disordered eating } \\
\text { behaviours) }\end{array}$ & $\begin{array}{l}\text { EAT-40: } 12,7 \% \\
\text { BULIT: } 9,7 \%\end{array}$ \\
\hline $\begin{array}{l}\text { Palma-Coca et al., } \\
2011^{32}\end{array}$ & 25.056 & $10-19$ & México & $\begin{array}{l}\text { The Brief Questionnaire } \\
\text { for Disordered Eating } \\
\text { Behaviors } \\
\text { (CBCAR) }\end{array}$ & $\begin{array}{l}\text { Comportamento } \\
\text { alimentar transtornado } \\
\text { (disordered eating } \\
\text { behaviour) }\end{array}$ & $4 \%$ \\
\hline Power et al., $2008^{33}$ & 723 meninas & $12-19$ & Quito, Equador & $\begin{array}{l}\text { Eating Attitudes Test } \\
\text { (EAT-26) } \\
\text { Eating Attitudes Test } \\
\text { (EAT-40) }\end{array}$ & $\begin{array}{l}\text { Atitudes alimentares } \\
\text { anormais (abnormal } \\
\text { eating attitudes) }\end{array}$ & $\begin{array}{l}\text { EAT-40: } 13 \% * \\
\text { EAT-26: } 14,4 \% *\end{array}$ \\
\hline Toro et al., $2006^{34}$ & $\begin{array}{l}467 \text { meninas } \\
\text { espanholas } \\
329 \text { meninas } \\
\text { mexicanas }\end{array}$ & $11-18$ & $\begin{array}{l}\text { Espanha e } \\
\text { México }\end{array}$ & $\begin{array}{l}\text { Eating Attitudes Test } \\
\text { (EAT-26) } \\
\text { The Eating Disorders } \\
\text { Assessment } \\
\text { Questionnaire (CETCA) }\end{array}$ & $\begin{array}{l}\text { Risco para TA (risk of } \\
\text { eating disorders) }\end{array}$ & $\begin{array}{l}24,6 \% \text { espanholas* } \\
22,5 \% \text { mexicanas* }\end{array}$ \\
\hline
\end{tabular}

Tabela 3 - Descrição de estudos realizados nos Estados Unidos e no Canadá sobre comportamentos de risco para transtornos alimentares em adolescentes

\begin{tabular}{|c|c|c|c|c|c|c|}
\hline Autor/Ano & $\begin{array}{l}\text { Populaçäo de } \\
\text { estudo }\end{array}$ & Idade (anos) & Estado/País & Metodologia & Nomenclatura utilizada & Prevalência/frequência* \\
\hline $\begin{array}{l}\text { Austin et al., } \\
2008^{35}\end{array}$ & 5.567 & 15,9 (média) & EUA & Eating Attitude Test (EAT-26) & $\begin{array}{l}\text { Comer transtornado } \\
\text { (disordered eating) }\end{array}$ & $\begin{array}{l}14,5 \% \text { meninas } \\
3,6 \% \text { meninos }\end{array}$ \\
\hline $\begin{array}{l}\text { Cook et al., } \\
2007^{36}\end{array}$ & 1.133 & $15-19$ & Canadá & $\begin{array}{l}\text { Centers for } \\
\text { Disease Control and Prevention's Youth Risk } \\
\text { Factor } \\
\text { Surveillance Instrument }\end{array}$ & $\begin{array}{l}\text { Comportamento } \\
\text { alimentar transtornado } \\
\text { (disordered eating } \\
\text { behaviour) }\end{array}$ & $\begin{array}{l}\text { Geral: } 16 \%{ }^{*} \\
\text { Grupo que se achava maior: } \\
\text { Jejum: } 15 \%{ }^{*} \\
\text { Remédios para emagrecer: } 5,6 \%^{*} \\
\text { Vômito autoinduzido: } 7,5 \%{ }^{*}\end{array}$ \\
\hline $\begin{array}{l}\text { D'Souza et al., } \\
2005^{37}\end{array}$ & 1.027 & $14-19$ & EUA & Eating Attitude Test (EAT-26) & $\begin{array}{l}\text { Sintomas de TA } \\
\text { (eating disorder } \\
\text { symptoms) }\end{array}$ & $\begin{array}{l}22 \% \text { meninas* }^{*} \\
2 \% \text { meninos }^{*}\end{array}$ \\
\hline $\begin{array}{l}\text { Doyle et al., } \\
2007^{38}\end{array}$ & 81 & $12-17$ & $\begin{array}{l}\text { San Diego e } \\
\text { St. Louis, EUA }\end{array}$ & $\begin{array}{l}\text { Eating Disorder Examination - } \\
\text { Questionnaire } \\
\text { (EDE-Q) }\end{array}$ & $\begin{array}{l}\text { Risco para TA } \\
\text { (risk for eatings } \\
\text { disorders) }\end{array}$ & $\begin{array}{l}\text { Purgação: 1,2\%* } \\
\text { Uso de remédios para emagrecer: } 3,7 \% \%^{*} \\
\text { Exercício físico excessivo: } 33,3 \%^{*} \\
\text { Compulsão: } 29,6 \%^{*}\end{array}$ \\
\hline $\begin{array}{l}\text { Haines et al., } \\
2010^{39}\end{array}$ & $\begin{array}{l}6.022 \\
\text { meninas } \\
\text { e } 4.518 \\
\text { meninos }\end{array}$ & $11-17$ & EUA & $\begin{array}{l}\text { Perguntas próprias sobre métodos } \\
\text { purgativos, compulsão alimentar e prática } \\
\text { de dietas restritivas }\end{array}$ & $\begin{array}{l}\text { Comer transtornado } \\
\text { (disordered eating) }\end{array}$ & $\begin{array}{l}1998 \\
\text { Purgação } \\
\text { Meninas: } 3,7 \% \\
\text { Meninos: 0,7\% } \\
\text { Compulsão } \\
\text { Meninas: 7,1\% } \\
\text { Meninos: 2,0\% } \\
\text { Follow-up (1999-2001) } \\
\text { Purgação } \\
\text { Meninas: 7,8\% } \\
\text { Compulsão } \\
\text { Meninas: 11,8\% } \\
\text { Meninos: 4,5\% }\end{array}$ \\
\hline $\begin{array}{l}\text { Haines et al., } \\
2011^{13}\end{array}$ & 5.740 & 16 (média) & EUA & Eating Attitudes Test (EAT-26) & $\begin{array}{l}\text { Risco de TA (risk of } \\
\text { eating disorders) }\end{array}$ & $\begin{array}{l}\text { Meninas: } 14,4 \% \\
\text { Meninos: } 3,8 \%\end{array}$ \\
\hline
\end{tabular}




\begin{tabular}{|c|c|c|c|c|c|c|}
\hline Autor/Ano & $\begin{array}{l}\text { População de } \\
\text { estudo }\end{array}$ & Idade (anos) & Estado/País & Metodologia & Nomenclatura utilizada & Prevalência/frequência* \\
\hline $\begin{array}{l}\text { Haley et al., } \\
2010^{40}\end{array}$ & 16.289 & $\begin{array}{l}\text { Série escolar: } \\
8^{\text {a }} \text { e } 11^{a}\end{array}$ & $\begin{array}{l}\text { Oregon } \\
\text { Counties, } \\
\text { EUA }\end{array}$ & $\begin{array}{l}\text { Questões próprias: comportamento não } \\
\text { saudável para perda de peso, para manter } \\
\text { ou evitar ganho de peso: jejum, vomitar, } \\
\text { usar laxantes, tomar remédios para } \\
\text { emagrecer sem consentimento médico }\end{array}$ & $\begin{array}{l}\text { Práticas não saudáveis } \\
\text { para perda de peso } \\
\text { (unhealthy weight loss } \\
\text { practices-UWLP) }\end{array}$ & $11,6 \%$ \\
\hline $\begin{array}{l}\text { Jonate } \\
\text { Birmingham, } \\
2004^{41}\end{array}$ & 396 & $12-19$ & Canadá & Eating Attitudes Test (EAT-26) & $\begin{array}{l}\text { Comer transtornado } \\
\text { (disordered eating) }\end{array}$ & $\begin{array}{l}\text { Meninas: } 17,3 \%^{*} \\
\text { Meninos: } 8,3 \%^{*}\end{array}$ \\
\hline $\begin{array}{l}\text { McVey et al., } \\
2004^{42}\end{array}$ & $\begin{array}{l}2.279 \\
\text { meninas }\end{array}$ & $10-14$ & $\begin{array}{l}\text { Ontario, } \\
\text { Canadá }\end{array}$ & Children's Eating Attitudes Test (ChEAT) & $\begin{array}{l}\text { Atitudes alimentares } \\
\text { negativas (negative } \\
\text { eating attitudes) }\end{array}$ & $10,5 \%$ \\
\hline $\begin{array}{l}\text { Neumark- } \\
\text { Sztainer et al., } \\
2006^{43}\end{array}$ & 2.516 & $12-20$ & $\begin{array}{l}\text { Minneapolis/ } \\
\text { St. Paul, EUA }\end{array}$ & $\begin{array}{l}\text { Questões próprias sobre frequência de } \\
\text { dieta no último ano e outros métodos } \\
\text { para perder ou manter peso no último ano } \\
\text { (dieta, jejum, remédios para emagrecer, } \\
\text { vômito autoinduzido, uso de laxantes) }\end{array}$ & $\begin{array}{l}\text { Comer transtornado } \\
\text { (disordered eating) }\end{array}$ & $\begin{array}{l}\text { Dieta restritiva: } \\
\text { Meninas: } 57 \% \\
\text { Meninos: } 25,3 \% \\
\text { Práticas não saudáveis para controle do } \\
\text { peso: } \\
\text { Meninas: } 57,8 \% \\
\text { Meninos: } 31,3 \%\end{array}$ \\
\hline $\begin{array}{l}\text { Neumark- } \\
\text { Sztainer et al., } \\
2009^{44}\end{array}$ & $\begin{array}{l}412 \\
\text { adolescentes } \\
\text { com } \\
\text { sobrepeso } \\
\text { (follow-up) }\end{array}$ & $12-20$ & $\begin{array}{l}\text { Minneapolis/ } \\
\text { St. Paul, EUA }\end{array}$ & $\begin{array}{l}\text { Questões próprias sobre compulsão } \\
\text { alimentar, métodos extremos para controle } \\
\text { do peso (remédios para emagrecer, vômito } \\
\text { autoinduzido, uso de laxantes e diuréticos) } \\
\text { e práticas não saudáveis para controle do } \\
\text { peso (jejum, comer muito pouca comida, } \\
\text { usar substitutos de alimentos e pular } \\
\text { refeições) }\end{array}$ & $\begin{array}{l}\text { Comer transtornado } \\
\text { (disordered eating) }\end{array}$ & $\begin{array}{l}(1998-1999): 30,8 \% \\
(2003-2004): 40,1 \%\end{array}$ \\
\hline $\begin{array}{l}\text { Pinhas et al., } \\
2008^{45}\end{array}$ & 868 & $13-20$ & $\begin{array}{l}\text { Toronto, } \\
\text { Canadá }\end{array}$ & Eating Attitudes Test (EAT-26) & $\begin{array}{l}\text { Comer transtornado } \\
\text { (disordered eating) }\end{array}$ & $\begin{array}{l}\text { Judias: } 25 \% \\
\text { Não judias: } 18 \%\end{array}$ \\
\hline $\begin{array}{l}\text { Pisetsky et al., } \\
2008^{46}\end{array}$ & 13.917 & $\begin{array}{l}\text { Série escolar: } \\
9^{\mathrm{a}-12^{\mathrm{a}}}\end{array}$ & EUA & $\begin{array}{l}\text { Questão própria: resposta positiva para } \\
\text { comportamentos para controlar o peso } \\
\text { (jejuar, tomar remédio para emagrecer, } \\
\text { vomitar e usar laxante) }\end{array}$ & $\begin{array}{l}\text { Comer transtornado } \\
\text { (disordered eating) }\end{array}$ & $\begin{array}{l}\text { Meninas: } 21,8 \% \\
\text { Meninos: } 11,2 \%\end{array}$ \\
\hline $\begin{array}{l}\text { The McKnight } \\
\text { Investigators, } \\
2003^{47}\end{array}$ & $\begin{array}{l}1.103 \\
\text { meninas }\end{array}$ & $\begin{array}{l}\text { Série escolar: } \\
\text { 6a-9a }\end{array}$ & $\begin{array}{l}\text { Califórnia, } \\
\text { EUA }\end{array}$ & $\begin{array}{l}\text { McKnight Eating Disorder Examination for } \\
\text { adolescents (com base no DSM-IV para } \\
\text { classificar TA clínicos ou subclínicos) }\end{array}$ & $\begin{array}{l}\text { Fatores de risco para TA } \\
\text { (risk factors for eating } \\
\text { disorder) }\end{array}$ & $\begin{array}{l}\text { Transtorno alimentar parcial ou completo: } \\
2,9 \%^{*}\end{array}$ \\
\hline $\begin{array}{l}\text { Waasdorp et } \\
\text { al., } 2007^{48}\end{array}$ & 340 & $12-21$ & EUA & Eating attitude test (EAT-26) & $\begin{array}{l}\text { Comer transtornado } \\
\text { (disordered eating) }\end{array}$ & $8 \% *$ \\
\hline
\end{tabular}

Tabela 4 - Descrição de estudos realizados em países da Europa, Ásia, África e Austrália sobre comportamentos de risco para transtornos alimentares em adolescentes

\begin{tabular}{|c|c|c|c|c|c|c|}
\hline Autor/Ano & $\begin{array}{l}\text { População de } \\
\text { estudo }\end{array}$ & Idade (anos) & País & Metodologia & Nomenclatura utilizada & Prevalência/Frequência* \\
\hline $\begin{array}{l}\text { Alonso et al., } \\
2005^{49}\end{array}$ & 2.483 & $12-18$ & Espanha & Eating Attitudes Test (EAT-40) & $\begin{array}{l}\text { Risco de desenvolver TA (risk of developing eating } \\
\text { disorders) }\end{array}$ & $\begin{array}{l}\text { Meninas: } 12,3 \% \\
\text { Meninos: } 3,2 \%\end{array}$ \\
\hline $\begin{array}{l}\text { Ballester et al., } \\
2002^{50}\end{array}$ & 1.025 & $14-19$ & $\begin{array}{l}\text { Girona, } \\
\text { Espanha }\end{array}$ & Eating attitude test (EAT-40) & $\begin{array}{l}\text { Risco potencial de transtornos do comportamento } \\
\text { alimentar } \\
\text { (potential risk of TCA) }\end{array}$ & $\begin{array}{l}\text { Meninas: } 16,3 \% \\
\text { Meninos: } 0,4 \%\end{array}$ \\
\hline $\begin{array}{l}\text { Beato-Fernández } \\
\text { et al., } 2004^{51}\end{array}$ & 1.076 & $13-15$ & Espanha & $\begin{array}{l}\text { Eating Attitudes Test (EAT-40) } \\
\text { Bulimic Investigatory Test } \\
\text { Edinburgh (BITE) }\end{array}$ & $\begin{array}{l}\text { Comportamento alimentar anormal (abnormal } \\
\text { eating behavior) }\end{array}$ & $3,7 \%^{*}$ \\
\hline $\begin{array}{l}\text { Díaz-Benavente } \\
\text { et al., } 2003^{52}\end{array}$ & 789 & 12 a 15 & $\begin{array}{l}\text { Sevília, } \\
\text { Espanha }\end{array}$ & Eating Attitude Test (EAT-40) & $\begin{array}{l}\text { Risco de TA } \\
\text { (risk of eating disorders) }\end{array}$ & $8,8 \%{ }^{*}$ \\
\hline $\begin{array}{l}\text { Boschi et al., } \\
2003^{53}\end{array}$ & 156 meninas & $14-18$ & Itália & Eating Disorder Inventory 2 (EDI-2) & $\begin{array}{l}\text { Transtorno alimentar não especificado - TANE } \\
\text { (eating disorders with particular attention to the } \\
\text { partial syndromes - EDNOS) }\end{array}$ & $\begin{array}{l}\text { BN: } 1,28 \%^{*} \\
\text { TCAP: } 1,28 \%^{*} \\
\text { TANE: } 10,25 \%^{*}\end{array}$ \\
\hline $\begin{array}{l}\text { Calado et al., } \\
2010^{54}\end{array}$ & 1.115 & $14-16$ & Espanha & Eating Attitude Test (EAT-26) & $\begin{array}{l}\text { Padrões alimentares transtornados (disordered } \\
\text { eating patterns) }\end{array}$ & $10,2 \%$ \\
\hline $\begin{array}{l}\text { Costarelli et al., } \\
2011^{55}\end{array}$ & 202 & $15-18$ & Grécia & Eating Attitude Test (EAT-26) & $\begin{array}{l}\text { Atitudes alimentares transtornadas (disordered } \\
\text { eating attitudes) }\end{array}$ & $18,3 \% *$ \\
\hline
\end{tabular}




\begin{tabular}{|c|c|c|c|c|c|c|}
\hline Autor/Ano & $\begin{array}{l}\text { População de } \\
\text { estudo }\end{array}$ & Idade (anos) & País & Metodologia & Nomenclatura utilizada & Prevalência/Frequência* \\
\hline $\begin{array}{l}\text { Cotrufo et al., } \\
2007^{56}\end{array}$ & 2.925 & $11-13$ & Itália & Eating Disorder Inventory-2 (EDI-2) & $\begin{array}{l}\text { Atitude alimentar transtornada e comportamento } \\
\text { alimentar anormal (eating disorder attitude and } \\
\text { abnormal eating behaviours) }\end{array}$ & $14 \% *$ \\
\hline $\begin{array}{l}\text { Domine et al., } \\
2009^{57}\end{array}$ & 3.890 meninos & $16-20$ & Suiça & $\begin{array}{l}\text { Questões próprias indicadoras } \\
\text { de DEB - disordered eating } \\
\text { behaviors: medo de ganhar peso, } \\
\text { insatisfação corporal após comer } \\
\text { muito, pensar sobre comida, } \\
\text { gostar da sensação de estômago } \\
\text { vazio, comer muito com perda de } \\
\text { controle e vômito autoinduzido }\end{array}$ & $\begin{array}{l}\text { Comportamento alimentar transtornado (disordered } \\
\text { eating behaviors) }\end{array}$ & $\begin{array}{l}\text { Preocupação com o peso } \\
\text { ou comportamento } \\
\text { alimentar não saudável: } \\
50 \%\end{array}$ \\
\hline $\begin{array}{l}\text { Eapen et al., } \\
2006^{58}\end{array}$ & 495 meninas & $13-18$ & $\begin{array}{l}\text { Emirados } \\
\text { Árabes } \\
\text { Unidos }\end{array}$ & Eating Attitudes Test (EAT-40) & $\begin{array}{l}\text { Atitudes alimentares transtornadas (disordered } \\
\text { eating attitudes) }\end{array}$ & $23,4 \% *$ \\
\hline $\begin{array}{l}\text { Fichter et al., } \\
2005^{59}\end{array}$ & $\begin{array}{l}N=2631 \\
(1.980) \\
N=2920 \\
(1.998)\end{array}$ & $13-19$ & $\begin{array}{l}\text { Grécia e } \\
\text { Alemanha }\end{array}$ & $\begin{array}{l}\text { Anorexia Nervosa Inventory for } \\
\text { Self-Rating (ANIS) }\end{array}$ & Distúrbios alimentares (eating disturbances) & $\begin{array}{l}\text { Anorexia nervosa } \\
\text { Meninas } \\
\text { Munique: } 0 \% * \\
\text { Veria: } 0,59 \%^{*} \\
\text { Bulimia nervosa } \\
\text { Munique: } 1,89 \%^{*} \\
\text { Veria: } 1,18 \% *\end{array}$ \\
\hline $\begin{array}{l}\text { Gandarillas et al., } \\
2004^{60}\end{array}$ & 4.334 & $15-18$ & Espanha & $\begin{array}{l}\text { Critérios do DSM-IV e Eating } \\
\text { Disorder Inventory (EDI) }\end{array}$ & Risco para TA (risk for eating disorders) & $\begin{array}{l}\text { Meninas: } 15,3 \% \\
\text { Meninos: } 2,2 \%\end{array}$ \\
\hline $\begin{array}{l}\text { Goñi } \\
\text { Grandmontagne } \\
\text { e Rodríguez } \\
\text { Fernández, } \\
2004^{61}\end{array}$ & 740 & $12-16$ & $\begin{array}{l}\text { Burgos, } \\
\text { Espanha }\end{array}$ & Eating Disorders Inventory (EDI) & $\begin{array}{l}\text { Transtornos do comportamento alimentar } \\
\text { (eating behavior disorders) }\end{array}$ & $21,2 \% *$ \\
\hline $\begin{array}{l}\text { González-Juárez } \\
\text { et al., 200762 }\end{array}$ & 2.195 & $12-18$ & Espanha & Eating Attitudes Test (EAT-26) & $\begin{array}{l}\text { Em risco de apresentar TA (en riesgo de presentar } \\
\text { trastornos de la alimentación) }\end{array}$ & $8,8 \%$ \\
\hline $\begin{array}{l}\text { Grange et al., } \\
2004^{63}\end{array}$ & 813 & $\begin{array}{l}16,8 \\
\text { (média) }\end{array}$ & África do Sul & Eating Attitudes Test (EAT-26) & Comer transtornado (disordered eating) & $38,3 \% *$ \\
\hline $\begin{array}{l}\text { Hautala et al., } \\
2008^{64}\end{array}$ & 372 & $15-17$ & Finlândia & $\begin{array}{l}\text { Sick, Control, } \\
\text { One, Fat, Food (SCOFF) } \\
\text { questionnaire }\end{array}$ & $\begin{array}{l}\text { Sintomas de TA } \\
\text { (eating disorders symptoms) }\end{array}$ & $12 \% *$ \\
\hline $\begin{array}{l}\text { Hautala et al., } \\
2008^{65}\end{array}$ & 1.036 & $14-15$ & Finlândia & $\begin{array}{l}\text { Sick, Control, } \\
\text { One, Fat, Food (SCOFF) } \\
\text { questionnaire }\end{array}$ & Comer transtornado (disordered eating) & $\begin{array}{l}\text { Meninas: } 24 \% * \\
\text { Meninos: } 16 \% \text { * }\end{array}$ \\
\hline $\begin{array}{l}\text { Herpertz- } \\
\text { Dahlmann et al., } \\
2008^{66}\end{array}$ & 1.895 & $11-17$ & Alemanha & $\begin{array}{l}\text { Sick, Control, } \\
\text { One, Fat, Food (SCOFF) } \\
\text { questionnaire }\end{array}$ & $\begin{array}{l}\text { Comportamentos e atitudes alimentares } \\
\text { transtornados (disordered eating behaviour and } \\
\text { attitudes) }\end{array}$ & $\begin{array}{l}\text { Meninas: } 29,4 \% \\
\text { Meninos: } 14,4 \%\end{array}$ \\
\hline $\begin{array}{l}\text { Isomaa et al., } \\
2010^{67}\end{array}$ & 128 & 15 & Finlândia & $\begin{array}{l}\text { Rating of Anorexia and Bulimia- } \\
\text { Teenager version (RAB-T) }\end{array}$ & $\begin{array}{l}\text { Risco de TA } \\
\text { (risk of eating disorders) }\end{array}$ & $\begin{array}{l}\text { Meninas: } 14,4 \% * \\
\text { Meninos: } 0,6 \% *\end{array}$ \\
\hline $\begin{array}{l}\text { Kaluski et al., } \\
2008^{68}\end{array}$ & 2.978 meninas & $11-19$ & Israel & $\begin{array}{l}\text { Questionário adaptado do Sick, } \\
\text { Control, } \\
\text { One, Fat, Food (SCOFF) } \\
\text { questionnaire }\end{array}$ & Comer transtornado (disordered eating) & $30 \%$ \\
\hline $\begin{array}{l}\text { Knez et al., } \\
2006^{69}\end{array}$ & 480 & $10-16$ & Croácia & $\begin{array}{l}\text { Children's Eating Attitude Test } \\
\text { (ChEAT-26) }\end{array}$ & $\begin{array}{l}\text { Comportamentos e atitudes alimentares } \\
\text { transtornados (disturbed eating attitudes and } \\
\text { behaviors) }\end{array}$ & $\begin{array}{l}\text { Comportamento } \\
\text { alimentar } \\
\text { moderadamente } \\
\text { transtornado: } 31 \% * \\
\text { Comportamento } \\
\text { alimentar severamente } \\
\text { transtornado: } 7,5 \%{ }^{*}\end{array}$ \\
\hline $\begin{array}{l}\text { Lal e Abraham, } \\
2011^{70}\end{array}$ & 461 meninas & $12-17$ & Delhi, India & $\begin{array}{l}\text { Quality of Life for Eating Disorders } \\
\text { questionnaire (QOLED) }\end{array}$ & $\begin{array}{l}\text { Comportamento alimentar - Eating behavior (EB) } \\
\text { Transtorno Alimentar - Eating disorder (ED) }\end{array}$ & $\begin{array}{l}\text { EB: } 1,2 \% * \\
\text { ED: } 1,1 \% *\end{array}$ \\
\hline $\begin{array}{l}\text { Latzer e } \\
\text { Tzischinsky, } \\
2003^{71}\end{array}$ & 1.316 meninas & $12-18$ & Israel & $\begin{array}{l}\text { Eating Disorder Inventory-2 (EDI-2) } \\
\text { com } 11 \text { subescalas }\end{array}$ & $\begin{array}{l}\text { Comportamentos e atitudes alimentares } \\
\text { transtornados (disordered eating behaviors and } \\
\text { attitudes) }\end{array}$ & $\begin{array}{l}\text { Adolescentes mais } \\
\text { velhas obtiveram escores } \\
\text { maiores no EDI-2 }\end{array}$ \\
\hline
\end{tabular}




\begin{tabular}{|c|c|c|c|c|c|c|}
\hline Autor/Ano & $\begin{array}{l}\text { População de } \\
\text { estudo }\end{array}$ & Idade (anos) & País & Metodologia & Nomenclatura utilizada & Prevalência//rrequência* \\
\hline $\begin{array}{l}\text { Latzer e } \\
\text { Tzischinsky, 200572 }\end{array}$ & 1.270 meninas & $12-18$ & Israel & Eating Attitude Test (EAT-26) & $\begin{array}{l}\text { Atitudes alimentares anormais } \\
\text { (abnormal eating attitudes) }\end{array}$ & $19,5 \%$ \\
\hline $\begin{array}{l}\text { Latzer et al., } \\
2007^{73}\end{array}$ & 1.131 meninas & $12-18$ & Israel & Eating Disorder Inventory-2 (EDI-2) & Comer transtornado (disordered eating) & $13 \%$ \\
\hline $\begin{array}{l}\text { Lobera et al., } \\
2009^{74}\end{array}$ & 841 & $12-19$ & Espanha & $\begin{array}{l}\text { Eating Attitudes Test (EAT-40) } \\
\text { Sick Control On Fat Food (SCOFF) }\end{array}$ & $\begin{array}{l}\text { Comportamento alimentar de risco (conductas } \\
\text { alimentarias de riesgo) }\end{array}$ & $\begin{array}{l}\text { Pontuação de risco: } \\
\text { SCOFF: } 21,29 \%^{*} \\
\text { EAT- } 40: 7,13 \%^{*}\end{array}$ \\
\hline $\begin{array}{l}\text { López-Guimera } \\
\text { et al., 2008 }\end{array}$ & 323 meninas & $\begin{array}{l}\text { 13,11 } \\
\text { (média) }\end{array}$ & $\begin{array}{l}\text { Catalonia, } \\
\text { Espanha }\end{array}$ & Eating Attitude Test (EAT-26) & $\begin{array}{l}\text { Atitudes alimentares transtornadas (disordered } \\
\text { eating attitudes) }\end{array}$ & $\begin{array}{l}14 \% \%^{*} \text { faziam dieta } \\
\text { e destas, } 70 \% * \\
\text { apresentavam } \\
\text { atitudes alimentares } \\
\text { desordenadas }\end{array}$ \\
\hline $\begin{array}{l}\text { Maor et al., } \\
2006^{15}\end{array}$ & 283 & $\begin{array}{l}\text { Série } \\
\text { escolar: } \\
7^{\mathrm{a}-12^{\mathrm{a}}}\end{array}$ & Israel & Eating Attitudes Test (EAT-26) & $\begin{array}{l}\text { Atitudes alimentares anormais } \\
\text { (abnormal eating attitudes) }\end{array}$ & $20,8 \% *$ \\
\hline Marlowe, $2005^{76}$ & 836 & $10-15$ & $\begin{array}{l}\text { Bermuda, } \\
\text { Reino Unido }\end{array}$ & $\begin{array}{l}\text { Bulimic Investigatory Test } \\
\text { Edinburgh (BITE) } \\
\text { Eating Attitudes Test (EAT-40) }\end{array}$ & $\begin{array}{l}\text { Atitudes alimentares anormais } \\
\text { (abnormal eating atitudes) }\end{array}$ & $\begin{array}{l}\text { BITE: 0,24\%* } \\
\text { EAT: } 7,3 \% *\end{array}$ \\
\hline $\begin{array}{l}\text { Mateos-Padorno } \\
\text { et al., } 2010^{77}\end{array}$ & 1.364 & $12-17$ & $\begin{array}{l}\text { Island of } \\
\text { Gran Canaria }\end{array}$ & Eating attitude test (EAT-40) & $\begin{array}{l}\text { Risco de TA } \\
\text { (risk of eating disorders) }\end{array}$ & $13,4 \% *$ \\
\hline $\begin{array}{l}\text { Miotto et al., } \\
2002^{78}\end{array}$ & 847 & $15-19$ & Itália & $\begin{array}{l}\text { Eating Attitudes Test (EAT-40) } \\
\text { Bulimic Investigatory Test } \\
\text { Edinburgh (BITE) }\end{array}$ & $\begin{array}{l}\text { Risco de TA } \\
\text { (risk of eating disorders) } \\
\text { Padrões alimentares anormais } \\
\text { (abnormal eating patterns) }\end{array}$ & $\begin{array}{l}\text { BITE: } \\
\text { Meninas: 4,1\% } \\
\text { Meninos: } 0,3 \% \\
\text { EAT: } \\
\text { Meninas: } 15,8 \% \\
\text { Meninos: 2,8\% }\end{array}$ \\
\hline $\begin{array}{l}\text { Miotto et al., } \\
2003^{16}\end{array}$ & 930 & $15-19$ & Itália & $\begin{array}{l}\text { Eating Attitudes Test (EAT-40) } \\
\text { Bulimic Investigatory Test } \\
\text { Edinburgh (BITE) }\end{array}$ & $\begin{array}{l}\text { Padrões alimentares anormais } \\
\text { (abnormal eating patterns) }\end{array}$ & $\begin{array}{l}\text { EAT } \\
\text { Meninas: } 15 \% \\
\text { Meninos: } 2,7 \% \\
\text { BITE } \\
\text { Meninas: } 4,2 \% \\
\text { Meninos: } 0,3 \%\end{array}$ \\
\hline $\begin{array}{l}\text { Miotto et al., } \\
2003^{79}\end{array}$ & 847 & $15-19$ & Itália & $\begin{array}{l}\text { Eating Attitudes Test (EAT-40) } \\
\text { Bulimic Investigatory Test } \\
\text { Edinburgh (BITE) }\end{array}$ & $\begin{array}{l}\text { Risco de TA } \\
\text { (risk of eating disorders) } \\
\text { Padrões alimentares anormais } \\
\text { (abnormal eating patterns) }\end{array}$ & $\begin{array}{l}\text { EAT } \\
\text { Meninas: 15,8\% } \\
\text { Meninos: 2,8\% } \\
\text { BITE } \\
\text { Meninas: 4,1\% } \\
\text { Meninos: 0,3\% }\end{array}$ \\
\hline $\begin{array}{l}\text { Muro-Sans e } \\
\text { Amador-Campos, } \\
2007^{80}\end{array}$ & 1.155 & $10-17$ & Espanha & $\begin{array}{l}\text { Eating Disorder Inventory-2 } \\
\text { (EDI-2) e uma entrevista clínica } \\
\text { estruturada (EDE-12) }\end{array}$ & Transtornos alimentares (eating disorders) & $\begin{array}{l}\text { EDI-2: } 8 \% \\
\text { EDI-2 + EDE-12: 1,28\% } \\
\text { (2,31\% das meninas e } \\
0,17 \% \text { dos meninos) }\end{array}$ \\
\hline $\begin{array}{l}\text { Murrin et al., } \\
2007^{81}\end{array}$ & 2.469 & $10-17$ & Irlanda & $\begin{array}{l}\text { IMC e percepção do tamanho } \\
\text { corporal. } \\
\text { Risco para TA: adolescentes com } \\
\left.\text { baixo peso (IMC }<18,5 \mathrm{~kg} / \mathrm{m}^{2}\right) \\
\text { e que pensavam estar muito } \\
\text { gordos. }\end{array}$ & $\begin{array}{l}\text { Risco para TA } \\
\text { (risk of eating disorders) }\end{array}$ & $10,7 \%$ \\
\hline $0^{\prime}$ Dea, $2009^{82}$ & 470 & $11-14$ & Australia & Eating Disorders Inventory (EDI) & $\begin{array}{l}\text { Risco de TA } \\
\text { (eating disorders risk) }\end{array}$ & $15,1 \% *$ em alto risco \\
\hline $\begin{array}{l}\text { Pamies-Aubalat } \\
\text { et al., } 2011^{83}\end{array}$ & 2.142 & $12-16$ & $\begin{array}{l}\text { Alicante, } \\
\text { Espanha }\end{array}$ & Eating Attitude Test (EAT-40) & $\begin{array}{l}\text { Comportamento de risco para desenvolver TA (risk } \\
\text { behaviours of developing an ED) }\end{array}$ & $11,2 \%$ \\
\hline $\begin{array}{l}\text { Preti et al., } \\
2006^{84}\end{array}$ & 828 & $15-19$ & Itália & $\begin{array}{l}\text { Eating Attitudes Test (EAT-40) } \\
\text { Bulimic Investigatory Test } \\
\text { Edinburgh (BITE) }\end{array}$ & Comer transtornado (disordered eating) & $\begin{array}{l}\text { Meninas: } 12,7 \% \\
\text { Meninos: } 2,8 \%\end{array}$ \\
\hline Soo et al., $2008^{85}$ & 489 meninas & $15-17$ & Malásia & $\begin{array}{l}\text { Restrained Eating scale of the } \\
\text { Dutch Eating Behaoiour } \\
\text { Questionnaire (RESDEBQ) } \\
\text { Binge Scale Questionnaire (BSQ) }\end{array}$ & $\begin{array}{l}\text { Comportamento alimentar } \\
\text { (eating behaviour) }\end{array}$ & $\begin{array}{l}\text { Dieta restritiva: } 36 \% \\
\text { Compulsão alimentar: } \\
35,4 \% \\
\quad \text { continuação }\end{array}$ \\
\hline
\end{tabular}




\begin{tabular}{|c|c|c|c|c|c|c|}
\hline Autor/Ano & $\begin{array}{l}\text { População de } \\
\text { estudo }\end{array}$ & Idade (anos) & País & Metodologia & Nomenclatura utilizada & Prevalência/Frequência* \\
\hline Tam et al., $2007^{86}$ & 2.382 & $10-21$ & Hong Kong & Eating attitude test (EAT-26) & Comer transtornado (disordered eating) & $\begin{array}{l}\text { Meninas: } 6,5 \% \\
\text { Meninos: } 3,9 \%\end{array}$ \\
\hline $\begin{array}{l}\text { Toselli et al., } \\
2005^{87}\end{array}$ & 2.146 & $14-19$ & Itália & $\begin{array}{l}\text { Inventory for the Screening of } \\
\text { Eating Disorders (ISED) }\end{array}$ & $\begin{array}{l}\text { Comportamento alimentar transtornado (disordered } \\
\text { eating behaviours) }\end{array}$ & $\begin{array}{l}\text { Dieta restritiva } \\
\text { Meninas: } 58,4 \% \\
\text { Meninos: } 27,6 \% \\
\text { Atividade física } \\
\text { Meninas: } 44,9 \% \\
\text { Meninos: } 27,8 \% \\
\text { Vômito autoinduzido } \\
\text { Meninas: } 6,3 \% \\
\text { Meninos: } 2,3 \% \\
\text { Laxantes } \\
\text { Meninas: } 2,7 \% \\
\text { Meninos: } 2 \%\end{array}$ \\
\hline $\begin{array}{l}\text { Veses et al., } \\
2011^{88}\end{array}$ & 195 & $13-18$ & Espanha & SCOFF questionnaire & $\begin{array}{l}\text { Risco de TA } \\
\text { (risk of eating disorders) }\end{array}$ & $\begin{array}{l}\text { Meninas: } 24,7 \% \text { * } \\
\text { Meninos: } 17,3 \% *\end{array}$ \\
\hline $\begin{array}{l}\text { Yanez et al., } \\
2007^{89}\end{array}$ & 969 meninas & $12-16$ & $\begin{array}{l}\text { Barcelona, } \\
\text { Espanha }\end{array}$ & Eating Attitude Test (EAT-26) & $\begin{array}{l}\text { Atitudes alimentares anormais } \\
\text { (abnormal eating attitudes) }\end{array}$ & $10,1 \%$ \\
\hline
\end{tabular}

Foram encontrados nos artigos localizados 27 diferentes termos ou conjunto de termos para caracterizar "comportamentos de risco para TA", variando entre comportamentos, atitudes, padrões, práticas, sintomas e risco de transtornos alimentares.

A nomenclatura mais frequente foi "risco de transtornos alimentares" ("risk of eating disorders"), utilizado em 16 estudos $(21,0 \%)$, seguido de "comer transtornado" ("disordered eating"), utilizado em 14 estudos (18,4\%). No contexto do Brasil, "comportamento de risco para transtorno alimentar" ("eating disorder risk behavior") foi utilizado em 3 (37,5\%) dos 8 estudos nacionais, sendo as outras maneiras de nomear comportamento de risco para TA utilizadas muito variadas.

Outros nomes também foram frequentes - "comportamento alimentar transtornado" ("disordered eating behaviors") e "atitudes alimentares transtornadas" ("disoredered eating attitudes") foram utilizados em 7 (9,2\%) estudos cada. Os termos "atitudes alimentares anormais" ("abnormal eating attitudes") e "comportamentos alimentares anormais" ("abnormal eating behaviors") foram utilizados em $5(6,6 \%)$ e 3 $(3,9 \%)$ estudos, respectivamente, além dos termos "padrões alimentares anormais" ("abnormal eating patterns") e "sintomas de transtornos alimentares" ("eating disorders symptoms"), que foram utilizados em $3(3,9 \%)$ estudos cada.

Observa-se, em todas as tabelas, que os pesquisadores utilizaram basicamente questionários validados ou desenvolvidos especificamente para a pesquisa como instrumentos para avaliar comportamento de risco para TA. Verificou-se que 65 (85,5\%) trabalhos avaliaram comportamento de risco para TA utilizando questionários padronizados e, em alguns casos, mais de um tipo de questionário foi utilizado no mesmo trabalho.

Dentre os que usaram questionários, 39 (51,3\%) utilizaram EAT (versão 26 ou 40), 10 (13,1\%) utilizaram o BITE, 9
$(11,8 \%)$ utilizaram EDI, $6(7,9 \%)$ utilizaram o Sick, Control, One, Fat, Food (SCOFF) questionnaire, 3 (3,9\%) utilizaram EDE-Q e 2 $(2,6 \%)$ utilizaram o Children's Eating Attitudes Test (ChEAT). Os outros questionários foram utilizados em apenas um estudo cada (1,3\%), sendo eles: BULIT, Anorexia Nervosa Inventory for Self-Rating (ANIS), Rating of Anorexia and Bulimia-Teenager version (RAB-T), The Eating Disorders Assessment Questionnaire (CETCA), Inventory for the Screening of Eating Disorders (ISED), The Brief Questionnaire for Disordered Eating Behaviors (CBCAR), Quality of Life for Eating Disorders questionnaire (QOLED), Restrained Eating scale of the Dutch Eating Behavior Questionnaire (RESDEBQ) e Binge Scale Questionnaire (BSQ).

Além disso, 12 (15,8\%) trabalhos fizeram uso de questões desenvolvidas pelos autores do estudo, que envolviam perguntas diretas sobre medo de ganhar peso e métodos para perder peso como vômito autoinduzido, uso de laxantes, diuréticos ou remédios para emagrecer; fazer jejum, comer pouca comida, usar substitutos alimentares, pular refeições, fumar mais cigarros e fazer dieta restritiva.

Entre os estudos nacionais, observou-se o predomínio dos questionários padronizados EAT-26 e BITE, e o primeiro foi utilizado por 5 estudos (62,5\%) e o segundo, por 4 (50\%), ambos tendo sido utilizados em conjunto em 2 trabalhos. Apenas um estudo nacional não utilizou questionário padronizado, optando por questões desenvolvidas e adaptadas por Hay $(1998)^{8}$ sobre compulsão, dieta restritiva, uso de laxante, diurético ou vômito autoinduzido.

A prevalência/frequência de comportamentos de risco para TA variou de $1,1 \%{ }^{10}$ a $39,04 \%{ }^{24}$ nos estudos nacionais (Tabela 1); de 0,8\% ${ }^{11}$ a 53,3\% ${ }^{30}$ na América Latina (Tabela 2); de $2 \%^{37}$ a $57,8 \%{ }^{43}$ nos estudos realizados nos Estados Unidos e no Canadá (Tabela 3); e nos estudos realizados na Europa, Ásia, África e Austrália (Tabela 4) a prevalência variou de $0,24 \%{ }^{76}$ a $58,4 \%{ }^{87}$. 
Observando-se a prevalência/frequência de comportamentos de risco segundo o tipo de questionário utilizado, verifica-se variação de $2 \%{ }^{37}$ a $38,3 \%{ }^{63}$ segundo o EAT-26; de $0,4 \%{ }^{50}$ a $23,4 \%{ }^{58}$ segundo o EAT-40; de $1,9 \%{ }^{30}$ a $57 \% \%^{43}$ utilizando-se perguntas próprias para avaliar comportamentos de risco para TA; de $0,24 \%{ }^{76}$ a $39,04 \%{ }^{24}$ segundo o BITE; de $2,2 \%{ }^{60}$ a $21,2 \%{ }^{61}$ segundo o EDl; e de $12 \%{ }^{64}$ a $30 \%{ }^{68}$ segundo o SCOFF.

\section{DISCUSSÃO}

A presente revisão objetivou caracterizar o que é denominado "comportamento de risco" para TA entre adolescentes por diferentes autores e encontrou que uma variedade de nomes e instrumentos são utilizados nos estudos originais sobre o tema.

Os principais termos usados para caracterizar a presença de comportamentos de risco para TA foram "risco de transtornos alimentares"13,27-30,34,38,52,60,67,77-79,81,82,88 e "comer transtornado" $135,39,41,43-46,48,63,65,68,73,84,86$. Mesmo assim, mais de $60 \%$ dos estudos utilizaram outras diversas nomenclaturas e, no contexto do Brasil, observa-se que também não há padronização dos termos. Dessa forma, um pesquisador que objetiva conhecer e comparar dados sobre essa temática encontra dificuldade na seleção dos trabalhos e na compreensão daquilo que os autores se propuseram a avaliar.

Destaca-se, ainda, que os instrumentos utilizados para avaliar a frequência ou prevalência de "risco de TA" e de "comer transtornado" variaram entre diferentes questionários, de padronizados a questões desenvolvidas especificamente para o estudo, havendo, portanto, distinção nos instrumentos de avaliação.

O EAT (tanto na versão 26 ou 40 questões) foi o principal instrumento utilizado para avaliar comportamento de risco para TA entre os estudos selecionados. Esse é possivelmente o instrumento de autopreenchimento mais utilizado na área dos TA para estudos de rastreamento e avaliações em populações clínicas, em várias línguas e culturas, dada a sua facilidade de aplicação, eficiência, economia e não necessidade de treinamento para administração $0^{6,90}$.

No entanto, esse instrumento foi desenvolvido inicialmente como um teste para diagnóstico de anorexia nervosa e, como não se adequou para o diagnóstico do distúrbio, passou a ser utilizado para detectar casos clínicos em população de alto risco e identificar indivíduos com preocupações anormais com alimentação e peso. Ele avalia sintomas de restrição alimentar e identifica comportamentos de dieta, os quais podem ou não estar associados a sintomas mais específicos de TA.

Como o EAT é uma escala que foi desenvolvida há muito tempo, alguns cuidados com a interpretação dos resultados tornam-se necessários. Deve-se questionar, por exemplo, se a resposta positiva à questão "eu como alimentos dietéticos" é, nos dias atuais, uma medida de comer transtornado; da mesma forma, para se responder que se conhece o valor calórico dos alimentos, em uma época de obrigatoriedade de rótulos alimentares ${ }^{91}$. Além dessas considerações, podem existir questões de tradução e diferenças de interpretações culturais². No Brasil, uma primeira tradução do teste foi disponibilizada em $1994^{92}$ e uma validação com adolescentes do sexo feminino foi publicada em 2004 ${ }^{93}$. A versão de Bighetti et al. ${ }^{93}$ encontrou alpha de Cronbach de 0,82 e a de Nunes et al.22 foi reavaliada em 2005 e teve consistência interna (alpha de Cronbach) de 0,75; essa reavaliação também mostrou baixos coeficientes de validade para o instrumento aplicado em um contexto do Brasil, concluindo que existem dúvidas sobre a capacidade do teste de identificar comportamentos alimentares anormais na população jovem feminina ${ }^{22}$.

Ainda sobre tradução, nenhuma das versões em português parece trazer corretamente o significado da afirmativa "I worry about the amount of calories in the food I eat". Foi traduzida por Nunes et al. ${ }^{92}$ como "sei quantas calorias têm os alimentos que como" e, por Bighetti et al.93, como "presto atenção à quantidade de calorias dos alimentos que eu como", considerando que um comportamento mais transtornado seria contar as calorias de tudo que se consome ${ }^{91}$.

Ademais, segundo Garfinkel e Newman ${ }^{94}$, o EAT tem boas propriedades psicométricas de confiabilidade e validade, razoável sensibilidade e especificidade para TA, mas valor preditivo positivo muito baixo, em virtude de os TA serem relativamente incomuns. Por isso, os autores afirmam que o EAT é válido para rastreamento de distúrbios da alimentação em geral, em população de risco e subgrupos específicos, e para comparações entre grupos e diferentes culturas. Observa-se, no entanto, pelos resultados encontrados nesta revisão, que a variabilidade nos resultados de frequência ou prevalência foi muito grande e, dadas as considerações anteriores, pode ser temerário fazer comparações entre grupos, apenas com o resultado do EAT, assim como pensar em maior ou menor frequência de risco de TA em determinado grupo, sem avaliações concomitantes e considerações amplas sobre o contexto socioeconômico e cultural, além de considerar-se a cultura alimentar e a cultura de "dieta" e de imagem corporal do grupo avaliado.

O segundo questionário mais utilizado para rastrear sintomas de TA foi o BITE, desenvolvido para o rastreamento e avaliação da gravidade da BN, e apresentando propriedades psicométricas adequadas no estudo original ${ }^{6}$. Esse instrumento fornece os resultados em duas escalas, uma de gravidade e outra de sintomas especíicos de BN, o que pode explicar menores frequências na comparação dos resultados de estudos que utilizaram o BITE e trabalhos que utilizaram outros questionários - mesmo assim, a amplitude dos resultados é grande. 
O EDI, terceiro instrumento mais utilizado, foi desenvolvido para avaliar características comportamentais e psicológicas da AN e BN. É composto por 64 itens em oito subescalas que avaliam a busca da magreza, bulimia, insatisfação corporal, perfeccionismo, falta de confiança, consciência corporal e medo da maturidade 6 . Vários estudos constataram sua validade e confiabilidade, e existem normas publicadas para aplicação em adolescentes, mas não há estudo de adaptação do EDI para o português ${ }^{95}$. No caso dos resultados de frequência ou prevalência com EDI, a amplitude foi menor do que com EAT e BITE. Observa-se, portanto, que é difícil comparar os resultados encontrados por meio desses questionários, uma vez que uma gama de características é avaliada, e não apenas ou especificamente os comportamentos específicos de TA, como restrição, compulsão e purgação.

Alguns fatores podem auxiliar na análise da grande variabilidade na prevalência e frequência de comportamentos de risco encontrada nos estudos: 1) diferentes comportamentos são avaliados por meio de cada instrumento; quando o estudo avaliou sintomas mais severos, encontrou prevalências menores, tal fato foi verificado em estudos que utilizaram o BITE, por exemplo, cujo resultado pode ser variado em diferentes graus de problema alimentar, de acordo com o escore do instrumento ${ }^{10,24} ; 2$ ) outro exemplo é a avaliação de práticas não saudáveis para controle do peso, que são comportamentos menos severos (comer pouca comida, pular refeição, usar substitutos de alimentos, tomar remédios para emagrecer e fumar mais cigarros) e apresentam maior prevalência43; 3) alguns estudos apresentaram seus resultados em relação à amostra completa e outros dicotomizaram segundo sexo ${ }^{37,50,60}$, aparentando prevalências menores; 4) outros estudos apresentam apenas resultados de comportamentos específicos como restrição alimentar ${ }^{30,87}$ ou sintomas bulímicos ${ }^{30}, \mathrm{o}$ que pode levar à apresentação de menores prevalências; 5) pode haver também superestimação dos comportamentos de risco devido a dificuldade de entendimento do questionário por parte do entrevistado, como no estudo com adolescentes sul-africanos ${ }^{63}$ que tiveram dificuldade de compreender o questionário em inglês e responderam positivamente a alguns itens do EAT, não por apresentarem preocupação com o peso, mas sim por práticas culturais (vomitar o alimento como ritual de limpeza para proteger o corpo de doenças) e circunstâncias socioeconômicas (após ficar com muita fome, comiam demais quando havia comida disponível e "tinham" que vomitar), caracterizando falsos-positivos.

Como exemplo do fator 2 listado acima, além dos sintomas clássicos de TA, outros comportamentos de risco menos graves para TA são avaliados por alguns autores que têm utilizado o termo "práticas não saudáveis para controle do peso" 43,44 para agrupar: fazer jejum ou comer muito pouca comida, pular refeições, usar substitutos de alimentos como suplementos ou shakes, tomar remédios para emagrecer ou fumar mais cigarros. Esses comportamentos contribuem para aumentar o risco de desenvolver transtornos alimentares clinicamente significantes e, portanto, também devem ser avaliados, mas não é adequado comparar sua prevalência com a de sintomas mais graves, específicos para TA, pois tais práticas tendem a ser mais prevalentes que os sintomas clássicos de TA ${ }^{43}$.

Um fator relevante das avaliações realizadas com questões focadas nos comportamentos específicos é que estas deveriam gerar resultados mais facilmente comparáveis. No entanto, a variabilidade ainda é razoável nos resultados obtidos de questões específicas elaboradas pelos autores sobre restrição alimentar: 24,7\% em estudo nacional ${ }^{7}, 53,3 \%$ na América Latina ${ }^{30}$ e $57 \%{ }^{43}$ em meninas, nos Estados Unidos; uso de laxantes, vômitos autoinduzidos e diuréticos: 5,1\% no Brasil $^{7}$ e 7,8\% nos Estados Unidos 39 ; e de compulsão alimentar: $37,3 \%$ no Brasil $^{7}$ e 11,8\% em meninas, nos Estados Unidos $^{39}$. Isso se deve, pelo menos em parte, ao fato de que esses questionários não geram escores, e, portanto, seus resultados são apresentados detalhando-se cada comportamento. Dessa forma, verifica-se maior prevalência de restrição alimentar, seguida de compulsão alimentar e menores prevalências de comportamentos compensatórios (uso de laxantes, vômitos autoinduzidos e diuréticos).

A vantagem de perguntas específicas relativas aos questionários longos seria a possibilidade de facilitar o entendimento por parte dos adolescentes. Questionários simplificados são, portanto, uma alternativa eficaz para rastrear o risco de desenvolver TA entre adolescentes?.

Dentre eles, destaca-se o SCOFF questionnaire, que consiste em cinco questões relativas às características fundamentais da $\mathrm{AN}$ e $\mathrm{BN}$, desenvolvido para rastrear mulheres adultas em risco de TA, podendo também ser utilizado com adolescentes ${ }^{36,64,66}$. Questões próprias foram desenvolvidas para alguns estudos: McKnight et al. ${ }^{47}$ elaboraram quatro questões, concluindo que foram eficazes para identificar $80 \%$ das adolescentes que poderiam desenvolver TA. No Brasil, Ferreira e Veiga ${ }^{7}$ adaptaram um questionário elaborado por Hay ${ }^{8}$, que foi baseado no Eating Disorder Examination $(E D E)^{96}$ e considerado o "padrão-ouro" para o rastreamento dos TA². Esse questionário é composto por duas perguntas: a primeira investiga a frequência de compulsão alimentar e a segunda, subdividida em quatro questões, investiga a frequência de métodos para controlar o ganho de peso ou possíveis mecanismos compensatórios à ingestão alimentar excessiva, tais como o comportamento purgativo (uso de laxantes, diuréticos ou vômitos autoinduzidos) e dieta muito restritiva ou jejum ${ }^{97}$.

Ferreira e Veiga ${ }^{97}$ avaliaram esse questionário para identificar comportamentos de risco para TA em adolescentes do Rio de Janeiro e encontraram boa confiabilidade para rastrear a frequência da realização de dietas restritivas e para investigar o uso de diuréticos, laxantes e vômitos autoinduzi- 
dos e confiabilidade moderada para avaliar a frequência dos episódios de compulsão alimentar. Os autores concluíram também que, pelo fato de ser bem mais curto e simples do que os demais, e mesmo assim abranger questões acerca dos principais comportamentos indicadores da presença de risco para o desenvolvimento de TA, esse questionário pode ser útil em estudos epidemiológicos para rastreamento do problema em jovens.

Além dos aspectos discutidos, sabe-se que vários fatores podem dificultar a caracterização do que é comportamento de risco para TA4; esses distúrbios podem se apresentar de maneira distinta em diferentes culturas. Por exemplo, critérios diagnósticos baseados em normas ocidentais podem não ser apropriados para países não ocidentais ${ }^{98}$; diferenças raciais e étnicas também são observadas ao se estudar a prevalência de psicopatologias em adolescentes ${ }^{99}$. E, de qualquer forma, deve-se atentar para a atual "globalização" de costumes alimentares e ideais de beleza ${ }^{100}$, que se disseminam pela mídia e que afetam até culturas distintas ${ }^{101}$.

Os presentes resultados chamam a atenção para a necessidade de padronização dos termos utilizados em pesquisas sobre risco de TA, assim como de padronização do método de avaliação do fenômeno, o que possibilitaria a comparação de dados dentro do mesmo país ou em diferentes locais e realidades. Reconhece-se também a necessidade de se definir melhor o que é comportamento disfuncional e o que é risco para o desenvolvimento do quadro.

Sugere-se que comportamentos de risco para transtornos alimentares poderiam ser caracterizados pela presença dos sintomas clássicos de TA, ou seja, restrição alimentar, compulsão ou purgação, e que as práticas menos graves para controle do peso poderiam ser denominadas "comer transtornado".

A padronização dos termos e metodologias de avaliação dos TA para identificação precoce desses comportamentos é essencial para o desenvolvimento de métodos de intervenção mais efetivos e para subsidiar ações de prevenção.

\section{CONCLUSÃO}

Foi encontrada ampla variação na maneira de caracterizar o comportamento de risco para TA, assim como nos tipos de instrumentos e nomenclaturas utilizados.

Os comportamentos de risco para TA foram caracterizados principalmente pela presença dos sintomas clássicos (restrição alimentar, purgação e compulsão), mas também foram associados a diferentes características alimentares, de peso e de imagem corporal.

O principal instrumento utilizado nos estudos foi o EAT e os termos mais frequentes foram "risco para transtornos alimentares" e "comer transtornado". A frequência de com- portamentos de risco para TA variou de acordo com o tipo de instrumento utilizado e com a região ou país de origem.

\section{CONTRIBUIÇÕES INDIVIDUAIS}

Greisse Viero da Silva Leal - Realizou a busca bibliográfica para concepção do artigo, assim como a análise dos resultados, discussão e conclusão.

Marle dos Santos Alvarenga - Contribuiu para o desenho metodológico do estudo, discussão e conclusão e revisou criticamente seu conteúdo intelectual.

Sonia Tucunduva Philippi - Revisou criticamente seu conteúdo intelectual.

Viviane Ozores Polacow e Táki Athanássios Cordás - Revisaram a versão final.

Todos os autores aprovaram a versão final a ser publicada.

\section{CONFLITOS DE INTERESSE}

Nenhum autor possui conflitos de interesse a serem declarados.

\section{REFERÊNCIAS}

1. American Dietetic Association (ADA). Position of the American Dietetic Association: nutrition intervention in the treatment of anorexia nervosa, bulimia nervosa, and other eating disorders. J Am Diet Assoc. 2006;106:2073-82.

2. Alvarenga MS, Scagliusi FB, Philippi ST. Comportamento de risco para transtorno alimentar em universitárias brasileiras. Rev Psiq Clín. 2011;38(1):3-7.

3. American Psychiatry Association (APA). Diagnostic and Statistical Manual of Mental Disorders (DSM-IV). 4a ed. Washington DC: American Psychiatry Association; 1994.

4. American Psychiatry Association (APA). Practice guideline for the treatment of patients with eating disorders. 3rd ed. Virginia: American Psychiatric Publishing; 2006.

5. Hay P. Epidemiologia dos transtornos alimentares: estado atual e desenvolvimentos futuros. Rev Bras Psiquiatr. 2002;24:13-7.

6. Freitas S, Gorenstein C, Appolinario JC. Instrumentos para a avaliação dos transtornos alimentares. Rev Bras Psiquiatr. 2002;24:34-8.

7. Ferreira JES, Veiga GV. Eating disorder risk behavior in Brazilian adolescents from low socio-economic level. Appetite. 2008;51:249-55.

8. Hay P. The epidemiology of eating disorder behaviors: an Australian community - based survey. J Eat Disord. 1998;23:371-82

9. Neumark-Sztainer D, Bauer KW, Friend S, Hannan PJ, Story M, Berge JM. Family weight talk and dieting: how much do they matter for body dissatisfaction and disordered eating behaviors in adolescent girls? J Adolesc Health. 2010;47:270-6.

10. Vilela JEM, Lamounier JA, Filho MAD, Neto JRB, Horta GM. Eating disorders in school children. J Pediatr (Rio J). 2004;80(1):49-54.

11. Barriguete-Meléndez JA, Unikel-Santoncini C, Aguilar-Salinas C, Córdoba-Villalobos JA, Shamah T, Barquera $S$, et al. Prevalence of abnormal eating behaviors in adolescents in Mexico (Mexican National Health and Nutrition Survey 2006). Salud Pub México. 2009;51(Suppl 4)

12. Bay DLB, Herscovici CR, Kovalskys I, Berner E, Orellana L, Bergesio LA. Alteraciones alimentarias en niños y adolescentes argentinos que concurren al consultorio del pediatra. Arch Argent Pediatr. 2005;103(4):305-16. 
13. Haines J, Ziyadeh Nj, Franko DI, MCDonald J, Mond Jm, Austin Sb. Screening high school students for eating disorders: validity of brief behavioral and attitudinal measures. J Sch Health. 2011;81:530-5.

14. Gomes JP, Legnani E, Legnani RFS, Gregório NP, Souza RK. Associação entre comportamento alimentar, consumo de cigarro, drogas e episódios depressivos em adolescentes. Rev Nutr. 2010; 23(5):755-62.

15. Maor NR, Sayag S, Dahan R, Hermoni D. Eating attitudes among adolescents. IMAJ. 2006:8:627-9.

16. Miotto P, De Coppi M, Frezza M, Preti A. Eating disorders and suicide risk factors in adolescents: an Italian community-based study. J Nerv Ment Dis. 2003;191:437-43.

17. Neumark-Sztainer D, Wall M, Story M, Fulkerson JA. Are family meal patterns associated with disordered eating behaviors among adolescents? J Adolesc Health. 2004;35:350-9.

18. Kelly SD, Howe CJ, Hendler JP, Lipman TH. Disordered eating behaviors in youth with type 1 diabetes. Diabetes Educ. 2005;34:572-83.

19. Neumark-Sztainer D, Wall MM, Haines JI, Story MT, Sherwood NE, Van den Berg PA. Shared risk and protective factors for overweight and disordered eating in adolescents. Am J Prev Med. 2007;33(5):359-69.

20. Alves E, Vasconcelos FAG, Calvo MCM, Neves J. Prevalência de sintomas de anorexia nervosa e insatisfação com a imagem corporal em adolescentes do sexo feminino do município de Florianópolis, Santa Catarina, Brasil. Cad Saude Publica. 2008;24(3):503-12.

21. Dunker KLL, Fernandes CPB, Filho DC. Influência do nível socioeconômico sobre comportamentos de risco para transtornos alimentares em adolescentes. J Bras Psiquiatr. 2009;58(3):156-61.

22. Nunes MA, Camey S, Olinto MTA, Mari JJ. The validity and 4-year test-retest reliability of the Brazilian version of the Eating Attitudes Test-26. Braz J Med Biol Res. 2005;38:1655-62.

23. Sampei MA, Sigulem DM, Novo NF, Juliano Y, Colugnati FAB. Eating attitudes and body image in ethnic Japanese and Caucasian adolescent girls in the city of São Paulo, Brazil. J Pediatr. 2009;85(2):122-8.

24. Souza-Kaneshima AM, França AA, Kneube DPF, Kaneshima EM. Identificação de distúrbios da imagem corporal e comportamentos favoráveis ao desenvolvimento da bulimia nervosa em adolescentes de uma Escola Pública do Ensino Médio de Maringá, Estado do Paraná. Acta Sci Health Sci. 2008;30(2):167-73.

25. Vale AMO, Kerr LRS, Bosi MLM. Risk behaviors for eating disorders among female adolescents from different social strata in the Brazilian Northeastern. Ciênc Saúde Coletiva. 2011:16(1):121-32

26. Souza MT, Silva MD, Carvalho R. Revisão integrativa: o que é e como fazer. Einstein. 2010;8(1 Pt 1):102-6.

27. Behar AR, Alviña WM, González CT, Rivera NA. Detección de actitudes y/o conductas predisponentes a trastornos alimentarios en estudiantes de enseñanza media de tres colegios particulares de viña del mar. Rev Chil Nutr. 2007;34(3).

28. Correa VML, Zubarew GT, Silva MP, Romero SMI. Prevalencia de riesgo de trastornos alimentarios en adolescentes mujeres escolares de la Región Metropolitana. Rev Chil Pediatr. 2006;77(2):153-60.

29. González MAM, Viveros GRO. Trastorno alimentario y su relación con la imagen corporal y la autoestima en adolescentes. Terapia Psicológica. 2009;27(2):181-90.

30. Granillo BAT, Jones-Rodriguez MPHG, Carvajal SC. Prevalence of eating disorders in Latina adolescents: associations with substance use and other correlates. J Adolesc Health. 2005;36:214-20.

31. Mancilla-Díaz JM, Lopez-Aguilar X, Franco-Paredes K, Alvarez-Rayon G, Vazquez-Arevalo $R$, Tellez-Giron MTO. Effect of gender and school level on disordered eating behaviours and attitudes in Mexican adolescents. Eat Weight Disord. 2009;14:e169-75.

32. Palma-Coca 0, Hernández-Serrato MI, Villalobos-Hernández A, Unikel-Santoncini C, OlaizFernández G, Bojorquez-Chapela I. Association of socioeconomic status, problem behaviors, and disordered eating in Mexican adolescents: results of the Mexican National Health and Nutrition Survey 2006. J Adolesc Health. 2011;49:400-6.

33. Power $P$, Power $L$, Canadas MB. Low socioeconomic status predicts abnormal eating attitudes in Latin American female adolescents. Eat Disord. 2008;16:136-45.

34. Toro J, Gomez-Peresmitre G, Sentis J, Vallés A, Casula V, Castro J, et al. Eating disorders and body image in Spanish and Mexican female adolescents. Soc Psychiatry Psychiatr Epidemiol. 2006;41:556-65.
35. Austin SB, Ziyadeh NJ, Forman S, Prokop LA, Keliher A, Jacobs D. Screening high school students for eating disorders: results of a national initiative. Prev Chronic Dis. 2008;5(4).

36. Cook SJ, MacPherson K, Langille DB. Far from ideal - Weight perception, weight control, and associated risky behaviour of adolescent girls in Nova Scotia. Can Fam Phys. 2007; $53: 678-84$

37. D'Souza CM, Forman SF, Austin SB. Follow-up evaluation of a high school eating disorders screening program: knowledge, awareness and self-referral. J Adolesc Health. 2005;36:208-13.

38. Doyle AC, Grange D, Goldschmidt A, Wilfley DE. Psychosocial and physical impairment in overweight adolescents at high risk for eating disorders. Obesity. 2007;15(1):145-54.

39. Haines J, Kleinman KP, Rifas-Shiman SL, Field AE, Austin SB. Examination of shared risk and protective factors for overweight and disordered eating among adolescents. Arch Pediatr Adolesc Med. 2010;164(4):336-43.

40. Haley CC, Hedberg K, Leman RF. Disordered eating and unhealthy weight loss practices: which adolescents are at highest risk? J Adolesc Health. 2010;47:102-5.

41. Jonat $L M$, Birmingham CL. Disordered eating attitudes and behaviours in the high-school students of a rural Canadian community. Eat Weight Disord. 2004;9(4):285-9.

42. McVey G, Tweed S, Blackmore E. Dieting among preadolescent and young adolescent females. CMAJ. 2004;170(10):1559-61

43. Neumark-Sztainer D, Wall M, Guo J, Story M, Haines J, Eisenberg M. Obesity, disordered eating, and eating disorders in a longitudinal study of adolescents: how do dieters fare 5 years later? J Am Diet Assoc. 2006;106:559-68.

44. Neumark-Sztainer D, Wall M, Story M, Sherwood NE. Five-year longitudinal predictive factors for disordered eating in a population-based sample of overweight adolescents: implications for prevention and treatment. Int J Eat Disord. 2009;42:664-72.

45. Pinhas L, Heinmaa M, Bryden P, Bradley S, Toner B. Disordered eating in Jewish adolescent girls. Can J Psychiatry. 2008;53(9):601-8.

46. Pisetsky EM, Chao YM, Dierker LC, May AM, Striegel-Moore RH. Disordered eating and substance use in high-school students: results from the youth risk behavior surveillance system. Int J Eat Disord. 2008:41:464-70.

47. The McKnight Investigators. Risk factors for the onset of eating disorders in adolescent girls: results of the McKnight longitudinal risk factor study. Am J Psychiatry. 2003;160:248-54.

48. Waasdorp CE, Caboot JB, Robinson CA, Abraham AA, Adelman WP. Screening military dependent adolescent females for disordered eating. Mil Med. 2007;172(9):962-7.

49. Alonso ATV, Rodríguez MAR, Alonso JEL, Carretero GR, Martín MF. Eating disorders. Prevalence and risk profile among secondary school students. Soc Psychiatry Psychiatr Epidemiol. 2005:40:980-7.

50. Ballester FD, De Gracia BM, Patiño MJ, Suñol Gurnés C, Ferrer AM. Eating attitudes and body satisfaction in adolescents: a prevalence study. Actas Esp Psiquiatr. 2002;30(4):207-12.

51. Beato-Fernández L, Rodríguez-Cano T, Belmonte-Llario A, Martínez-Delgado C. Risk factors for eating disorders in adolescents. A Spanish community-based longitudinal study. Eur Child Adolesc Psychiatry. 2004;13:287-94.

52. Díaz-Benavente M, Rodríguez MF, Martín LC, Hiruela Benjumea MV. Risk factors related with eating disorders in a community of adolescents. Aten Primaria. 2003;32(7):403-7.

53. Boschi V, Siervo M, D'Orsi P, Margiotta N, Trapanese E, Basile F, et al. Body composition, eating behavior, food-body concerns and eating disorders in adolescent girls. Ann Nutr Metab. 2003;47(6):284-93.

54. Calado M, Lameiras M, Sepúlveda AR, Rodrıquez Y, Carrera MV. The mass media exposure and disordered eating behaviours in Spanish secondary students. Eur Eat Disord Rev. 2010;18:417-27.

55. Costarelli V, Antonopoulou K, Mavrovounioti C. Psychosocial characteristics in relation to disordered eating attitudes in Greek adolescents. Eur Eat Disord Rev. 2011;19:322-30.

56. Cotrufo P, Cella S, Cremato F, Labella AG. Eating disorder attitude and abnormal eating behaviours in a sample of 11-13-year-old school children: the role of pubertal body transformation. Eat Weight Disord. 2007;12(4):154-60.

57. Domine F, Berchtold A, Akre C, Michaud PA, Suris JC. Disordered eating behaviors: what about boys? J Adolesc Health. 2009;44:111-7.

58. Eapen V, Mabrouk AA, Bin-0thman S. Disordered eating attitudes and symptomatology among adolescent girls in the United Arab Emirates. Eat Behav. 2006;7:53-60.

59. Fichter MM, Quadflieg N, Georgopoulou E, Xepapadakos F, Fthenakis EW. Time trends in eating disturbances in young Greek migrants. Int J Eat Disord. 2005;38:310-22. 
60. Gandarillas A, Febrel C, Galán I, León C, Zorrilla B, Bueno R. Population at risk for eating disorders in a Spanish region. Eat Weight Disord. 2004;9(3):179-85.

61. Goñi Grnadmontagne A, Rodríguez Fernández A. Eating disorders, sport practice and physical self-concept in adolescents. Actas Esp Psiquiatr. 2004;32(1):29-36.

62. González-Juárez C, Pérez-Pérez E, Cabrera BM, Paua IM, Pablo RR, Escalera PVT. Detección de adolescentes en riesgo de presentar trastornos de la alimentación. Aten Primaria. 2007;39(4):189-94

63. Grange D, Louw J, Breen A, Katzman MA. The meaning of 'self-starvation' in impoverished black adolescents in South Africa. Cult Med Psych. 2004;28:439-61.

64. Hautala L, Junnila J, Helenius H, Väänänen AM, Liuksila PR, Räihä $H$, et al. Adolescents with fluctuating symptoms of eating disorders: a 1-year prospective study. JAN. 2008;62(6):674-80.

65. Hautala LA, Junnila J, Helenius H, Väänänen AM, Liuksila PR, Räihä $H$, et al. Towards understanding gender differences in disordered eating among adolescents. J Clin Nurs. 2008:17:1803-13

66. Herpertz-Dahlmann B, Wille N, Holling H, Vloet TD, Ravens-Sieberer U; BELLA study group. Disordered eating behaviour and attitudes, associated psychopathology and health-related quality of life: results of the BELLA study. Eur Child Adolesc Psychiatry. 2008;17(Suppl 1):82-91.

67. Isomaa AL, Isomaa R, Marttunen M, Kaltiala-Heino R. Obesity and eating disturbances are common in 15-year-old adolescents. A two-step interview study. Nord J Psychiatry. 2010;64(2):123-9.

68. Kaluski DN, Natamba BK, Goldsmith R, Shimony T, Berry EM. Determinants of disordered eating behaviors among Israeli adolescent girls. Eat Disord. 2008;16:146-59.

69. Knez R, Munjas R, Petrovecki M, Paucic-Kirincic E, Peršic M. Disordered eating attitudes among elementary school population. J Adolesc Health. 2006;38:628-30.

70. Lal M, Abraham S. Adolescent development and eating disorder related quality of life in Indian females. Eating Weight Disord. 2011;16:e56-60

71. Latzer Y, Tzischinsky 0 . Weight concern, dieting and eating behaviors. A survey of Israeli high school girls. Int J Adolesc Med Health. 2003;15(4):295-305.

72. Latzer Y, Tzischinsky 0. Eating attitudes in a diverse sample of Israeli adolescent females: a comparison study. J Adolesc. 2005;28:317-23.

73. Latzer Y, Tzischinsky 0, Azaiza F. Disordered eating related behaviors among Arab schoolgirls in Israel: an epidemiological study. Int J Eat Disord. 2007;40:263-70.

74. Lobera IJ, Candau JR, Ríos PB, Berriatúa CM, Jaramillo RD, González MTM, et al. Conducta alimentaria e imagen corporal en una muestra de adolescentes de Sevilla. Nutr Hosp. 2009:24(5):568-73

75. López-Guimera G, Fauquet J, Portell M, Sanchez-Carracedo D, Raich RM. Dieting in Spanish adolescent girls. Eur Eat Disord Rev. 2008;16:234-40.

76. Marlowe K. A preliminary study of eat and bite scores for one school year in Bermuda: increased early anorexic measures related to socio-economic factors. Int I Soc Psychiatry. 2005:51(1):5-12

77. Mateos-Padorno C, Scoffier S, Polifrone M, Martínez-Patiño MJ, Martínez-Vidal A, Sánchez MLZ. Analysis of eating disorders among 12-17 year-old adolescents in the island of Gran Canaria. Eat Weight Disord. 2010;15(3):e190-4.

78. Miotto P, De Coppi M, Frezza M, Rossi M, Preti A. Social desirability and eating disorders. A community study of an Italian school-aged sample. Acta Psychiatr Scand. 2002;105:372-7.

79. Miotto P, De Coppi M, Frezza M, Preti A. The spectrum of eating disorders: prevalence in an area of Northeast Italy. Psychiatry Res. 2003;119:145-54.

80. Muro-Sans P, Amador-Campos JA. Prevalence of eating disorders in a Spanish community adolescent sample. Eat Weight Disord. 2007;12(1):1-6.
81. Murrin C, McNicholas F, Keogh L, Shiely F, Corrigan H, Gabhainn SN, et al. Correlates of increased risk of eating disorders in Irish school children. Ir Med J. 2007;100(8):40-2.

82. O'Dea JA. Self perception score from zero to ten correlates well with standardized scales of adolescent self esteem, body dissatisfaction, eating disorders risk, depression, and anxiety. Int J Adolesc Med Health. 2009;21(4):509-17.

83. Pamies-Aubalat L, Marcos YQ, Castaño MB. Study of risk of eating disorders in a representative sample of adolescents. Med Clin. 2011;136(4):139-43.

84. Preti A, Pinna C, Nocco S, Mulliri E, Pilia S, Petretto DR, et al. Body of evidence: tattoos, body piercing, and eating disorder symptoms among adolescents. J Psychosom Res. 2006;61:561-6

85. Soo KL, Shariff ZM, Taib MNM. Eating behavior, body image and self-esteem of adolescent girls in Malaysia. Percept Mot Skills. 2008;106:833-44.

86. Tam CKM, Cherry FN, Yu CM, Young BWY. Disordered eating attitudes and behaviours among adolescents in Hong Kong: prevalence and correlates. J Paediatr Child Health. 2007:43:811-7

87. Toselli AL, Villani S, Ferro AM, Verri A, Cucurullo L, Marinoni A. Eating disorders and their correlates in high school adolescents of Northern Italy. Epidemiol Psichiatr Soc. 2005;14(2):91-9.

88. Veses AM, Martínez-Gómez D, Gómez-Martínez S, Zapatera B, Veiga ÓL, Marcos A. Association between excessive body fat and eating-disorder risk in adolescents: the AFINOS Study. Med Clin (Barc). 2011;136(14):620-2.

89. Yanez AA, Peix MA, Atserias N, Arnau A, Brug J. Association of eating attitudes between teenage girls and their parents. Int I Soc Psychiatry. 2007;53(6):507-13.

90. Mintz LB, O'Halloran MS. The Eating Attitudes Test: validation with DSM-IV eating disorders criteria. J Pers Assess. 2000;74:489-503.

91. Alvarenga MS, Scagliusi FB, Philippi ST. Development and validity of the Disordered Eating Attitude Scale (DEAS). Percept Mot Skills. 2010;110(2):379-95.

92. Nunes MA, Bagatini L, Abuchaim AL, Kunz A, Ramos D, Silva JA, et al. Considerações sobre 0 Teste de Atitudes Alimentares (EAT-26). Rev ABP-APAL. 1994;16:7-10.

93. Bighetti F, Santos CB, Santos JE, Ribeiro RPP. Tradução e validação do Eating Attitudes Test em adolescentes do sexo feminino de Ribeirão Preto-SP. J Bras Psiquiatr. 2004;53:339-46.

94. Garfinkel PE, Newman A. The eating attitudes test: twenty-five years later. Eat Weight Disord 2001:6(1):1-24.

95. Moya T, Scagliusi FB. Instrumentos de avaliação em transtornos alimentares. In: Alvarenga MS, Scagliusi FB, Philippi ST, organizadoras. Nutrição e transtornos alimentares. Barueri, SP: Manole; 2011

96. Fairburn CG, Beglin SJ. Assessment of eating disorder: interview or self-report questionnaire? Int J Eat Dis. 1994;16:363-70.

97. Ferreira JES, Veiga GV. Confiabilidade (teste-reteste) de um questionário simplificado para triagem de adolescentes com comportamentos de risco para transtornos alimentares em estudos epidemiológicos. Rev Bras Epidemiol. 2008;11(3):393-401.

98. Makino M, Tsuboi K, Dennerstein L. Prevalence of eating disorders: a comparison of Western and Non-Western Countries. Med Gen Med. 2004;6(3):49.

99. McLaughlin KA, Hilt LM, Nolen-Hoeksema S. Racial/Ethnic differences in internalizing and externalizing symptoms in adolescents. J Abnorm Child Psychol. 2007;35(5):801-16.

100. Alvarenga MS, Dunker KLL, Philippi ST, Scagliusi FB. Influência da mídia em universitárias brasileiras de diferentes regiões. J Bras Psiquiatr. 2010;59(2):111-8.

101. Becker AE, Fay KE, Agnew-Blais J, Khan AN, Striegel-Moore RH, Gilman SE. Social network media exposure and adolescent eating pathology in Fiji. BJP. 2011;198:43-50 